\title{
The ATLASGAL survey: The sample of young massive cluster progenitors ${ }^{\star}$
}

\author{
T. Csengeri ${ }^{1}$, S. Bontemps ${ }^{2}$, F. Wyrowski ${ }^{1}$, S. T. Megeath ${ }^{3}$ F. Motte ${ }^{4,5}$, A. Sanna ${ }^{1}$, M. Wienen ${ }^{1}$, and K. M. Menten ${ }^{1}$ \\ ${ }^{1}$ Max Planck Institute for Radioastronomy, Auf dem Hügel 69, 53121 Bonn, Germany \\ e-mail: ctimea@mpifr-bonn.mpg.de \\ 2 OASU/LAB-UMR5804, CNRS, Université Bordeaux 1, 33270 Floirac, France \\ 3 Ritter Astrophysical Research Center, Department of Physics and Astronomy, University of Toledo, 2801 W. Bancroft St., Toledo, \\ OH 43606, USA \\ ${ }^{4}$ Institut de Planétologie et d'Astrophysique de Grenoble, Univ. Grenoble Alpes - CNRS-INSU, BP 53, 38041 Grenoble Cedex 9 , \\ France \\ 5 Laboratoire AIM Paris Saclay, CEA-INSU/CNRS-Université Paris Diderot, IRFU/SAp CEA-Saclay, 91191 Gif-sur-Yvette, France
}

Received 4 February 2016 / Accepted 16 December 2016

\begin{abstract}
Context. The progenitors of high-mass stars and clusters are still challenging to recognise. Only unbiased surveys, sensitive to compact regions of high dust column density, can unambiguously reveal such a small population of particularly massive and cold clumps. Aims. Here we use the ATLASGAL survey to identify a sample of candidate progenitors of massive clusters in the inner Galaxy. Methods. We characterise a flux limited sample of compact sources selected from the ATLASGAL survey. Sensitive mid-infrared data at 21-24 $\mu \mathrm{m}$ from the WISE and MIPSGAL surveys were explored to search for embedded objects, and complementary spectroscopic data were used to investigate their stability and their star formation activity.

Results. We identify an unbiased sample of infrared-quiet massive clumps in the Galaxy that potentially represent the earliest stages of massive cluster formation. An important fraction of this sample consists of sources that have not been studied in detail before. We first find that clumps hosting more evolved embedded objects and infrared-quiet clumps exhibit similar physical properties in terms of mass and size, suggesting that the sources are not only capable of forming high-mass stars, but likely also follow a single evolutionary track leading to the formation of massive clusters. The majority of the clumps are likely not in virial-equilibrium, suggesting collapse on the clump scale.

Conclusions. We identify the precursors of the most massive clusters in the Galaxy within our completeness limit, and argue that these objects are undergoing large-scale collapse. This is in line with the low number of infrared-quiet massive clumps and earlier findings that star formation, in particular for high-mass objects is a fast, dynamic process. We propose a scenario in which massive clumps start to fragment and collapse before their final mass is accumulated indicating that strong self-gravity and global collapse is needed to build up rich clusters and the most massive stars.
\end{abstract}

Key words. surveys - stars: massive - stars: formation

\section{Introduction}

The formation of massive stars and clusters remains an enigma in modern astrophysics. To explain the origin of high-mass stars, two theoretical scenarios are commonly invoked: the turbulentcore model (McKee \& Tan 2003) and a more dynamic view with cluster formation (Bonnell et al. 2001; Bonnell \& Bate 2006). The observational challenge to these theories is a systematic, Galaxy-wide search for the earliest phases of high-mass stars and massive protoclusters where the initial conditions can best be studied.

With "earliest phases" we refer to the starless/pre-stellar, and the subsequent phase where the submillimetre emission is still dominated by the massive core. This implies that no luminous massive young stellar object (MYSO) has yet emerged, and no ionising emission from embedded UC-H II regions. This phase is difficult to observe, however, mainly because of the

* Full Table 4 is only available at the CDS via anonymous ftp to cdsarc.u-strasbg.fr (130.79.128.5) or via

http://cdsarc.u-strasbg.fr/viz-bin/qcat?]/A+A/601/A60 short duration of less than $7.5 \times 10^{4} \mathrm{yr}$ at the scale of $\sim 0.3 \mathrm{pc}$ (Csengeri et al. 2014), and also because of the high complexity of active sites of high-mass star formation. Only a few candidates of massive starless cores have been claimed so far in the literature with masses up to $60 M_{\odot}$ in a size of $\simeq 0.1 \mathrm{pc}$ (Tan et al. 2013; Cyganowski et al. 2014), although towards the former object outflow activity has recently been discovered (Tan et al. 2016), and the absence of any molecular emission towards the latter object casts doubts on its Galactic origin. Confirmed examples of massive pre-stellar cores, as well as exploration of the even higher mass regime, are of extreme importance in order to constrain star formation models.

Early searches for the birthplaces of high-mass stars focused on IRAS selected infrared-bright sources, where low- and highluminosity sources were defined (Molinari et al. 1996). Highluminosity sources were associated with $\mathrm{UC}-\mathrm{H}$ II regions, i.e. the final stage of high-mass star formation, while the lower luminosity sources were associated with MYSOs. UC-H II regions have typical bolometric luminosities of $>10^{4} L_{\odot}$, while $(\mathrm{M})$ YSOs show a broad distribution peaking between $3 \times 10^{3}-2 \times 10^{4} L_{\odot}$ 
(e.g. Molinari et al. 2000; Mottram et al. 2011). In fact, UC$\mathrm{H}$ II regions and lower luminosity sources fill a distinct space in a mass-luminosity diagram, strongly suggesting an evolutionary trend of more luminous objects with similar masses being more evolved than their lower luminosity counterparts (see Molinari et al. 1996; Sridharan et al. 2002; Elia et al. 2013).

Only with the advance of sensitive and large format detector arrays have (sub)millimetre surveys of entire molecular clouds and larger areas of the Galaxy revealed lower luminosity objects associated with very massive gas reservoirs (e.g. Motte et al. 2003， 2007; Mookerjea et al. 2004; López et al. 2011; Russeil et al. 2010; Tackenberg et al. 2012; Traficante et al. 2015; Svoboda et al. 2016). Systematic searches for the massive but colder and lower luminosity sources have begun. In an unbiased survey, Motte et al. (2007) has identified a sample of massive dense cores (MDCs) with masses between 40-200 $M_{\odot}$, sizes of $\sim 0.1 \mathrm{pc}$ and low luminosities $\left(<10^{3} L_{\odot}\right)$ in the Cygnus- $X$ region. Their high concentration of mass and low bolometric luminosity suggest that they host high-mass protostars, potential precursors to MYSOs. High angular-resolution observations of Bontemps et al. (2010) indeed identified a handful of individual high-mass protostars within MDCs with envelope masses in the range of $8-50 M_{\odot}$. Studies of the outflow activity further suggest that these protostars are the high-mass equivalents of Class 0 protostars (Duarte-Cabral et al. 2013). Altogether, this is strong evidence that low luminosity MDCs host objects in the earliest stages of high-mass protostellar evolution. An unbiased, systematic study to identify such objects on Galactic scales is the necessary next step for studying the initial conditions for high-mass star and cluster formation.

In this paper we present the first systematic and unbiased study of the brightest submillimetre sources in the inner Galaxy selected from the $870 \mu \mathrm{m}$ APEX Telescope LArge Survey of the GALaxy (ATLASGAL, Schuller et al. 2009; Csengeri et al. 2014); we estimate their physical properties and search for the most massive clumps still in an early evolutionary stage among them. This allows us to identify and study the properties of the youngest precursors of massive clusters in the Galaxy which potentially host high-mass protostars. Various samples have been studied from the ATLASGAL survey, mostly based on associations with $\mathrm{H}$ II regions and sources from the Red MSX Survey (RMS, Lumsden et al. 2013), and their flux densities at $8 \mu \mathrm{m}$ (GLIMPSE, Benjamin et al. 2003), and $24 \mu \mathrm{m}$ (MIPSGAL, Carey et al. 2009). Focusing on the Ist Galactic quadrant, Csengeri et al. (2016a) present the largest sample to date of 430 clumps in different evolutionary stages over a wide mass range. The sample studied in Giannetti et al. (2014) represents a subsample of this source selection limited to the hundred brightest sources in different evolutionary stages in both the Ist and the IVth Galactic quadrant. This strategic selection of ATLASGAL clumps over various evolutionary stages has been targeted by extensive spectroscopic follow-up observations; see details in Csengeri et al. (2016a). Other samples using selective star formation tracers of more evolved objects, such as clumps with $\mathrm{H}$ II regions, RMS sources, and associations with masers from the Methanol MultiBeam survey, have been discussed in a series of papers by Urquhart et al. (2013a,b, 2014).

The paper is organised as follows. In Sect. 2 we describe the flux limited sample of the brightest submillimetre sources from the ATLASGAL survey and investigate their statistical properties in Sect. 3. Using complementary $21-24 \mu \mathrm{m}$ data, we identify a sample of massive clumps which are potentially the youngest massive objects known so far. In Sect. 4 we study their physical properties and discuss their potential to form high-mass stars.

\section{Brightest submillimetre clumps from the ATLASGAL survey}

The ATLASGAL survey (Schuller et al. 2009) imaged the Galactic plane between Galactic longitude $\ell= \pm 60^{\circ}$ and Galactic latitude $b= \pm 1.5^{\circ}$ at $870 \mu \mathrm{m}$ with the LABOCA camera (Siringo et al. 2009) on the APEX Telescope (Güsten et al. 2006). In an extension of the survey, the $-80^{\circ}<\ell<-60^{\circ}$ and $-2^{\circ}<b<+1^{\circ}$ region was also mapped. Thus, all together the ATLASGAL survey imaged 420 sq. deg. of the Galactic plane at a 19'.2 spatial resolution. The catalogue of compact sources is $97 \%$ complete down to $\sim 260 M_{\odot}$ at the distance of the Galactic centre, i.e., $8 \mathrm{kpc}$, and $1 M_{\odot}$ at $500 \mathrm{pc}$ for cold clumps (Csengeri et al. 2014).

We study here a flux limited sample of the ATLASGAL compact sources within the highest sensitivity part of the survey, corresponding to a Galactic longitude range of $|\ell| \leq 60^{\circ}$. The initial sample was selected using a $5 \mathrm{Jy}$ beam-averaged flux density threshold, which translates to $\sim 650 M_{\odot}$ (see Csengeri et al. 2014) at an average distance of ATLASGAL sources of $d \simeq$ $4.5 \mathrm{kpc}$ (Wienen et al. 2015). We estimate the corresponding column density by

$N\left(\mathrm{H}_{2}\right)=\frac{F_{v} R}{B_{v}\left(T_{\mathrm{d}}\right) \Omega \kappa_{v} \mu_{\mathrm{H}_{2}} m_{\mathrm{H}}}\left[\mathrm{cm}^{-2}\right]$,

where $F_{v}$ is the beam-averaged peak flux density; $\Omega$ is the solid angle of the beam calculated by $\Omega=1.13 \times \Theta^{2}$, where $\Theta$ is $19^{\prime \prime} .2$; $\mu_{\mathrm{H}_{2}}$ is the mean molecular weight per hydrogen molecule and is equal to 2.8 (Kauffmann et al. 2008); $m_{\mathrm{H}}$ is the mass of a hydrogen atom; and $R$ corresponds to the gas-to-dust ratio of 100 . Our flux density threshold thus corresponds to a column density $\left(N_{\mathrm{H}_{2}}\right)$ of $4.9-40.8 \times 10^{23} \mathrm{~cm}^{-2}$ using a centre frequency of $345 \mathrm{GHz}$ at our resolution of $19^{\prime \prime} .2$, and dust temperatures of $10-40 \mathrm{~K}$. For the highest column density sources this translates to an extremely high visual extinction of $A_{\mathrm{V}}>521 \mathrm{mag}^{1}$ (see details in Sect. 2.3).

From the ATLASGAL compact source catalogue we find a total of 219 sources above the 5 Jy beam-averaged flux density threshold (Csengeri et al. 2014). In the subsequent steps we first provide distance estimates (Sect. 2.1), and then determine their physical properties (Sects. 2.2 and 2.3). Using midinfrared data between $21-24 \mu \mathrm{m}$ from the WISE point source catalogue (Wright et al. 2010) and photometry based on the MIPSGAL survey (Carey et al. 2009) and complemented with classification from the MYSO and UC-H II regions from the RMS (Urquhart et al. 2007) and CORNISH surveys (Hoare et al. 2012; Purcell et al. 2013), we then estimate the evolutionary stage based on the luminosity of the embedded protostars associated with the dust peaks (Sects. 2.4 and 2.5). This leads to the identification of infrared-quiet massive clumps in Sect. 2.6. In Sect. 2.7 we present molecular line data to characterise their associations with shocks from outflow activity to probe ongoing star formation.

\subsection{Distances and association with massive molecular complexes}

The large statistics of the ATLASGAL survey revealed that most of the compact sources, and correspondingly most of the star formation activity is associated with giant molecular complexes (GMCs; e.g. Csengeri et al. 2014). As a first step, therefore, we

1 For the conversion we use $A_{\mathrm{V}}=N_{\mathrm{H}_{2}} / 0.94 \times 10^{21} \mathrm{~cm}^{-2}$ (Bohlin et al. 1978). 
Table 1. Association of the brightest ATLASGAL sources with GMCs.

\begin{tabular}{|c|c|c|}
\hline $\begin{array}{l}\text { Associated GMC or } \\
\text { molecular cloud }\end{array}$ & $\begin{array}{l}d \\
{[\mathrm{kpc}]}\end{array}$ & $\begin{array}{l}\text { Number } \\
\text { of sources }\end{array}$ \\
\hline W51 & $5.41^{b}$ & 8 \\
\hline W49 & $11.11^{c}$ & 3 \\
\hline IRDC G34.4+0.2 & $1.56^{p}$ & 3 \\
\hline W43 & $5.49^{d}$ & 4 \\
\hline W31 & $4.95^{f}$ & 3 \\
\hline W33 & $2.4^{e}$ & 3 \\
\hline M17 & $1.98^{m}$ & 4 \\
\hline G5.89 & $1.28^{r}$ & 2 \\
\hline Galactic centre & $8.5^{j}$ & 9 \\
\hline NGC 6334 & $1.35^{i}$ & 9 \\
\hline RCW 122 & $1.8^{i, j}$ & 4 \\
\hline RCW 108-109 & $1.8^{a, l}$ & 3 \\
\hline G333.1-0.4 & $4.2^{a, g}$ & 6 \\
\hline RCW106 & $4.2^{a}$ & 5 \\
\hline G327 & $2.5^{a}$ & 3 \\
\hline RCW 95 & $2.5^{a, g, y}$ & 5 \\
\hline RCW 92 & $3.2^{a}$ & 2 \\
\hline G305 & $3.5^{q}$ & 2 \\
\hline G10.47 & $8.47^{f}$ & 1 \\
\hline RCW 120 & $1.3^{a}$ & 1 \\
\hline RCW 131/NGC 6537 & $1.7^{h}$ & 1 \\
\hline G345.5+1.0 & $1.8^{a, s}$ & 1 \\
\hline RCW 116b & $1.8^{a, g}$ & 1 \\
\hline RCW 117 & $1.8^{a, k}$ & 1 \\
\hline RCW 99 & $2.5^{a}$ & 1 \\
\hline G9.62+0.19 & $5.15^{n}$ & 1 \\
\hline G351.77 & $1.0^{\circ}$ & 1 \\
\hline SDC335 & $3.8^{a, \ddagger}$ & 1 \\
\hline
\end{tabular}

Notes. ${ }^{(a)}$ This work; ${ }^{(b)}$ Sato et al. (2010); (c) Zhang et al. (2013); (d) Zhang et al. (2014); ${ }^{(e)}$ Immer et al. (2013); (f) Sanna et al. (2014); ${ }^{(g)}$ Moisés et al. (2011); ${ }^{(y)}$ we revised the spectrophotometric distance of $1.81 \mathrm{kpc}$ by Moisés et al. (2011); ${ }^{(h)}$ Wu et al. (2014); ${ }^{(i)} \mathrm{Wu}$ et al (2012); ${ }^{(j)}$ Reid et al. (2014); ${ }^{(k)}$ Dutra et al. (2003); (l) Straw et al (1987); ${ }^{(m)}$ Xu et al. (2011); ${ }^{(n)}$ Sanna et al. (2009); ${ }^{(o)}$ Leurini et al. (2011); ${ }^{(p)}$ Kurayama et al. (2011); ${ }^{(q)}$ Russeil (2003); ${ }^{(r)}$ Motogi et al. (2011); ${ }^{(s)}$ López et al. (2011); ${ }^{(\ddagger)}$ Peretto et al. (2013) adopts $3.25 \mathrm{kpc}$.

associated the sample with known GMCs (see Table 1). In fact, our selection corresponds to the brightest submillimetre sources in the inner Galactic plane, and it covers well-characterised massive star-forming complexes such as Sgr B2, W51 Main, W49A, $\mathrm{W} 33$, and W43. Other examples are found at the edge of expanding bubbles like G348.1825+0.4829, which is the brightest compact source in the vicinity of RCW 120 (Zavagno et al. 2010). We find a few examples of relatively isolated sources, which are not associated with any known GMCs, but smaller molecular clouds, such as G0.5464-0.8521, G333.0164+0.7654, and G43.7950-0.1270, towards which our distance estimates may be less reliable than for the majority of the sample.

We collected distance estimates from the literature using primarily maser parallax measurements (see Reid et al. 2014, for an overview) or spectrophotometric distance estimates (e.g. Russeil 2003; Moisés et al. 2011) for the known GMCs. For the remaining unassociated sources we used the high-density tracer $\mathrm{NH}_{3}$ to determine kinematic distances from Wienen et al. (2012, 2015).
In short, the rest velocity of the source is compared to the Galactic rotation curve using the parameters derived by Reid et al. (2009) for the Ist and Brand \& Blitz (1993) for the IVth Galactic quadrants, respectively. We attempt to solve the distance ambiguity by making use of $\mathrm{HI}$ emission and $21 \mathrm{~cm}$ continuum absorption against bright $\mathrm{H}_{\mathrm{II}}$ regions (see Wienen et al. 2015, for details). We then visually inspected near-IR extinction maps (Schneider et al. 2010) and data from the Galactic Ring Survey (GRS, Jackson et al. 2006) to look for spatial and kinematic associations with nearby molecular clouds also considering the larger scale Galactic structure such as spiral arms, and associations with extinction features as well. We also inspected sensitive mid-infrared data from GLIMPSE and MIPSGAL to look for possible associations with infrared-dark dust lanes appearing in high contrast absorption at mid-infrared wavelengths.

In total we associate $78(36 \%)$ sources with available maser parallax measurements. In the Ist Galactic quadrant, such measurements are available for 59 out of 85 sources (70\%), providing a robust estimate of their physical parameters. Sources in the IVth Galactic quadrant have less reliable distance measurements owing to the lack of currently available maser parallax distances, except for G339.8841-1.2568 (Krishnan et al. 2015). We were able to provide distance estimates to all but one source in the sample (see Table 4). For simplicity, in the following analysis we exclude the 9 sources associated with the Galactic centre, leaving a total of 210 sources in our sample. An overview of their Galactic distribution is shown in Fig. 1.

\subsection{Temperature estimates}

Our sample includes clumps in various evolutionary stages such as active star-forming sites with clusters of known (UC-)H II regions (e.g. W51 Main, W49 N, G5.89) and typical hot molecular cores (HMCs; e.g. G10.47+0.3, G34.26+0.15) associated with infrared-bright MYSOs. Over such a large star formation activity, the estimated physical parameters from the $870 \mu \mathrm{m}$ dust emission can be highly sensitive to the used dust temperature. As a simple first-order approximation, a single dust temperature is commonly used for which a $T_{\mathrm{d}}$ of $18 \mathrm{~K}$ is a reasonable choice (see e.g. Csengeri et al. 2014) based on the Herschel measured dust temperatures dominated by the interstellar radiationfield (Bernard et al. 2010). However, the brightest submillimetre sources are more likely to host embedded heating source(s), which could imply higher dust temperatures. This is supported by statistical studies; for instance, Battersby et al. (2011) show that tracers of star formation are more likely associated with higher dust temperatures, while König et al. (2017) show an increase in dust temperature with evolutionary stage by fitting the spectral energy distribution of ATLASGAL clumps over a range of evolutionary stages. Hence, a temperature correction is necessary for clumps hosting UC-H II regions and infrared-bright protostars, and we discuss below our choice of a higher dust temperature.

At the high densities probed by the ATLASGAL survey it is reasonable to assume that the gas and dust are thermalised. Therefore, to investigate which dust temperature is appropriate for the warmer clumps, we can also rely on complementary molecular line data available in the literature. The $\mathrm{NH}_{3}$ measurements by Wienen et al. (2012) give a gas kinetic temperature between $12-35 \mathrm{~K}$ for a large sample of ATLASGAL sources within a beam of $\sim 40^{\prime \prime}$. Similarly, Dunham et al. (2011) derive a mean gas kinetic temperature of $17 \mathrm{~K}$ for a sample of 199 sources from the Bolocam Galactic Plane Survey (BGPS) with a beam of $31^{\prime \prime}$. This corresponds well to the average dust 


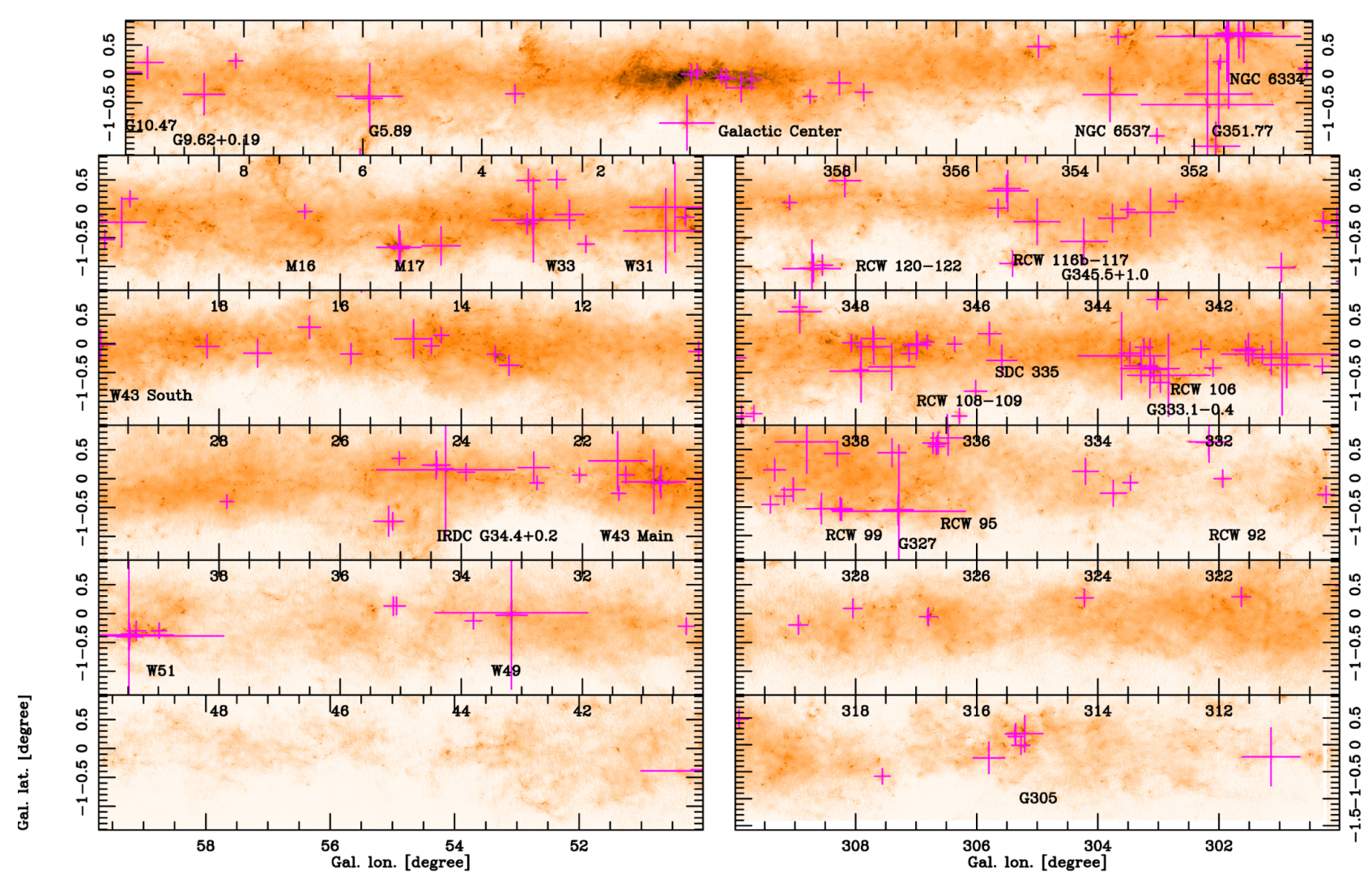

Fig. 1. Overview of the Galactic distribution of the sample and the associated molecular clouds. Figure adopted from Csengeri et al. (2016b) showing the ATLASGAL-Planck combined view of the inner galaxy. Magenta crosses show the location of the sources. The size of the symbols scales linearly with the peak flux density.

temperature also used in various studies of ATLASGAL selected sources (e.g. Csengeri et al. 2016a).

A total of 127 sources (corresponding to $58 \%$ of the sample) have kinetic temperature estimates from Wienen et al. (2012) and Wienen et al. (2017), and the bulk of these temperature measurements range between 17 and $35 \mathrm{~K}$. Since these measurements do not cover the full sample, they only allow us to estimate a reasonable temperature correction. Furthermore, as these studies mostly rely on the $(1,1)$ to $(2,2)$ inversion transitions of $\mathrm{NH}_{3}$, they are more sensitive to the cold gas rather than the hot gas component. Therefore, for the more evolved objects these temperatures should be considered as lower limits on the dust temperature, and thus an upper limit on the mass. When discussing the statistical properties of the sample (Sect.3), as a second-order approximation, we account for the increasing temperature towards sources with embedded massive stars using a higher $T_{\mathrm{d}}$ of $30 \mathrm{~K}$. This is consistent with the typical values used so far of 15-20 K for cold sources and up to 30-40 K for warm sources (e.g. Motte et al. 2007; Russeil et al. 2010).

\subsection{Estimating physical parameters: mass, size, density, and surface density}

We use the same formula as described in Schuller et al. (2009) and Csengeri et al. (2014) to estimate the gas mass from the optically thin $870 \mu \mathrm{m}$ dust emission

$M_{\mathrm{gas}}=\frac{S_{v} R d^{2}}{B_{v}\left(T_{\mathrm{d}}\right) \kappa_{v}} \simeq \frac{M_{\mathrm{gas}}}{\left[M_{\odot}\right]}=C \times \frac{S_{v}}{[\mathrm{Jy}]} \times\left(\frac{d}{[\mathrm{kpc}]}\right)^{2}$,

where $S_{v}$ is the integrated flux and $d$ corresponds to the distance of the sources. The numerical constant, $C_{18 \mathrm{~K}}=6.33$ for $v=345 \mathrm{GHz}, T_{\mathrm{d}}=18 \mathrm{~K}$, and $\kappa_{v}=0.0185 \mathrm{~cm}^{2} \mathrm{~g}^{-1}$ from Ossenkopf \& Henning (1994) accounting for a gas-to-dust ratio $(R)$ of 100 . We note that recent studies suggest a higher gas-todust ratio of 150 (Draine 2011), which would directly increase our mass estimates by 50\%. Peretto et al. (2013) adopt a 50\% lower $\kappa_{v}$, which would also lead to an increase in the estimated mass. Following the analysis in Sect. 2.2, we introduce a temperature corrected mass estimate $\left(M_{\text {corr }}\right)$ for the more evolved and thus likely warmer objects adopting $T_{\mathrm{d}}=30 \mathrm{~K}$, corresponding to $C_{30 \mathrm{~K}}=3.08$ from Sect. 3 .

An additional factor of uncertainty in the mass determination originates from optically thick free-free emission which may contribute to the $870 \mu \mathrm{m}$ flux density towards the clumps hosting $\mathrm{UC}-\mathrm{H}_{\mathrm{II}}$ regions. For a uniform density $\mathrm{H}$ II region this contribution can be negligible because the thermal dust emission dominates the spectral energy distribution (Hunter et al. 2000). However, for a non-uniform density source with high temperature and density, a more significant contribution from free-free emission is possible (e.g. Beuther et al. 2004; Keto 2002, 2003). This may lead to an overestimate of the mass. Schuller et al. (2009), however, estimate this contribution to be considerably lower than the thermal emission from dust at $345 \mathrm{GHz}$, not more than $20 \%$ for the most extreme cases. In addition, the already formed ionising sources are still compact and therefore are expected to have a minor influence on the mass estimates. To conclude, for sources associated with known embedded UC-H II regions we conservatively consider the derived masses as upper limits (Table 4).

In Csengeri et al. (2014) the ATLASGAL sources have been fitted with a 2D Gaussian, from which we estimate the source size following the method of Bontemps et al. (2010). We define the source radius $\left(R_{90}\right)$ which contains $90 \%$ of the total 
mass, assuming a 2D Gaussian profile, by $R_{90}=1.95 \times$ $F W H M_{\text {deconvolved }} / \sqrt{8 \ln 2}$, where $F W H M_{\text {deconvolved }}$ corresponds to the deconvolved source size ${ }^{2}$. From the mass and size, we use the following equations to calculate the mean volume density and surface density:

$$
\begin{gathered}
\frac{\bar{n}}{\left[\mathrm{~cm}^{-3}\right]}=\frac{M_{\mathrm{gas}}}{\frac{4}{3} R_{90}^{3} \pi} \\
\frac{\Sigma}{\left[\mathrm{g} \mathrm{cm}^{-2}\right]}=\frac{M_{\mathrm{gas}}}{R_{90}^{2} \pi} .
\end{gathered}
$$

The estimated physical parameters are listed in Table 4.

\subsection{Mid-infrared tracers of embedded protostars}

Here we investigate the properties of the embedded YSOs closely associated with the dust peaks in order to reveal their star formation activity and, in particular, the youngest clumps within the sample. To classify an embedded source as a high-mass young stellar object, we rely on the IRAS-based colour properties for embedded high-mass stars from Wood \& Churchwell (1989) following the method of Motte et al. (2007) and Russeil et al. (2010). Based on stellar models we adopt a luminosity threshold corresponding to objects with $L_{\text {bol }}>10^{4} L_{\odot}$, which potentially host a $>15 M_{\odot}$ zero age main sequence (ZAMS) star with a spectral type earlier than B0.5 (see also Mottram et al. 2011). In practice, using the expression from Motte et al. (2007), we translate this luminosity to a flux density threshold of $\sim 289 \mathrm{Jy}$ at $21 \mu \mathrm{m}$ flux for an object at $1 \mathrm{kpc}$. This limit is a factor of ten higher than was used for CygnusX (Motte et al. 2007) and NGC 6334 (Russeil et al. 2010), and we evaluate the implication of the higher luminosity limit in Sect. 2.5. We extrapolate between the $22 \mu \mathrm{m}$ and $24 \mu \mathrm{m}$ data assuming blackbody radiation for the embedded protostar following Russeil et al. (2010).

Below this luminosity threshold we find high-mass protostars or their potential precursors, which are still deeply embedded in their mass reservoir, while their energetics is dominated by accretion processes. Although theoretical models are strongly dependent on the accretion rates, they predict Class 0-like highmass protostars up to $L_{\text {bol }}<10^{4} L_{\odot}$ with an envelope mass of $>50 M_{\odot}$. Our threshold is also consistent with evolutionary tracks of high-mass stars assuming constant or varying accretion with rates of $10^{-4}-10^{-3} M_{\odot} \mathrm{yr}^{-1}$ (Duarte-Cabral et al. 2013; Kuiper \& Yorke 2013). In fact, the potentially highest mass protostar in Cygnus-X, N63, has an envelope mass of $\sim 44 M_{\odot}$ and $L_{\text {bol }} \sim 339 L_{\odot}$ estimated from SED models using Herschel data (Duarte-Cabral et al. 2013).

To extract the mid-infrared flux density of the sample, we first use the most sensitive and highest angular-resolution data from the MIPSGAL survey (Carey et al. 2009). The MIPS detector saturates at $2 \mathrm{Jy}$ for point sources, and confusion due to nearby point sources or bright extended emission may hinder the photometry at the position of the dust peaks. Considering only the point source saturation limit, the MIPSGAL survey reveals B3 type (or earlier) stars only beyond $3.8 \mathrm{kpc}$. Less luminous but closer objects are likely to appear saturated in the MIPS images. Therefore, in order to cover a continuous sensitivity range it is necessary to complement the MIPSGAL data with data from the WISE satellite. The PSF photometry of the WISE point source catalogue is sensitive up to a $~ 330 \mathrm{Jy}$ flux density threshold (Cutri et al. 2012).

\footnotetext{
2 We use 19".2 for the resolution of the ATLASGAL maps.
}

The MIPSGAL survey at $24 \mu \mathrm{m}$ has a $3 \sigma$ point source sensitivity of $2 \mathrm{mJy}$ at a $6^{\prime \prime}$ angular resolution, while the WISE survey provides a $5 \sigma$ point source sensitivity of $6 \mathrm{mJy}$ at a $12^{\prime \prime}$ resolution at $22 \mu \mathrm{m}$ in unconfused regions of the Galaxy. Owing to extended emission from nebulosity and polycyclic aromatic hydrocarbons (PAHs), these sensitivity levels may be higher towards confused regions. The factor of 4 difference in beam areas may also result in different flux densities determined for the same source from the MIPSGAL and WISE data. In Appendix A we discuss the cross-calibration between the MIPS and WISE photometry.

Although ultimately the sensitivity threshold is only a factor of few better for the MIPSGAL survey, its angular-resolution $\left(6^{\prime \prime}\right)$, which is more than three times higher, is a key benefit in our study. This angular resolution corresponds to a physical scale of $\sim 0.14 \mathrm{pc}$ at a $4.5 \mathrm{kpc}$ distance, which is the size scale of massive dense cores embedded in massive clumps (Motte et al. 2007; Zhang et al. 2009; Csengeri et al. 2011b). This angular resolution allows us to resolve and distinguish between infrared-bright and -quiet massive dense cores for objects located at distances closer than $\sim 4.5 \mathrm{kpc}$.

All sources were therefore first visually inspected using the publicly available calibrated data tiles from the MIPSGAL survey, and where no point source was found within $6^{\prime \prime}$ of the ATLASGAL peak position, we extracted an upper limit on the flux density (for more details see Appendix A.1). Aperture photometry was performed on point sources where no saturated pixels are present (Appendix A.2). Where the MIPSGAL data was not useable, we used the flux density from the WISE point source catalogue Wright et al. (2010, Appendix A.3) ${ }^{3}$.

In Fig. 2 we show two examples of massive clumps with no (or weak) embedded mid-infrared sources, the upper panel showing the WISE $22 \mu \mathrm{m}$ image of G340.9698-1.0212 and the lower panel showing the MIPSGAL $24 \mu \mathrm{m}$ image towards G34.43+00.24 (Garay et al. 2004; Rathborne et al. 2005); the rest of the sample is shown in Appendix B, Fig. B.1. This particular example shows the importance of angular resolution because this region hosts an already formed UC-H II region in the vicinity of G34.4+0.2-MM1 and MM2, while the brightest dust peak itself is clearly shifted from the position of the UC-H II region (Rathborne et al. 2005). The other embedded dust peaks in this clump are active sites of high-mass star formation in their earliest phase (Rathborne et al. 2008).

In Fig. 3, we show the resulting photometry measurements versus clump mass using $T_{\mathrm{d}}=18 \mathrm{~K}$ (see Sect. 2.3). The photometry is scaled to a common wavelength of $22 \mu \mathrm{m}$ and to a distance of $1 \mathrm{kpc}$. Using a homogeneous dust temperature facilitates the comparison with the larger sample of sources discussed in Sect. 2.5. While the brightest mid-infrared sources correspond to active high-mass star-forming sites in the inner Galaxy, we reveal a population of massive clumps which are clearly fainter at mid-infrared wavelengths (see also Sect. 2.6).

\subsection{Tracers of embedded high-mass stars}

According to stellar evolution models, stars of types B3B0.5 may also have luminosities between $L_{\mathrm{bol}}=10^{3}-10^{4} L_{\odot}$, hence they fall below our threshold introduced in Sect.2.4. We assess their potential contamination of the sample of the youngest massive clumps by using sensitive radio-surveys such as CORNISH (Hoare et al. 2012; Purcell et al. 2013) and

3 The WISE $22 \mu \mathrm{m}$ flux density is calculated from the W4 band magnitudes by using the formula $F_{22 \mu \mathrm{m}}=8.2839 \times 10^{\mathrm{mag}_{\mathrm{w}} /-2.5}$. 

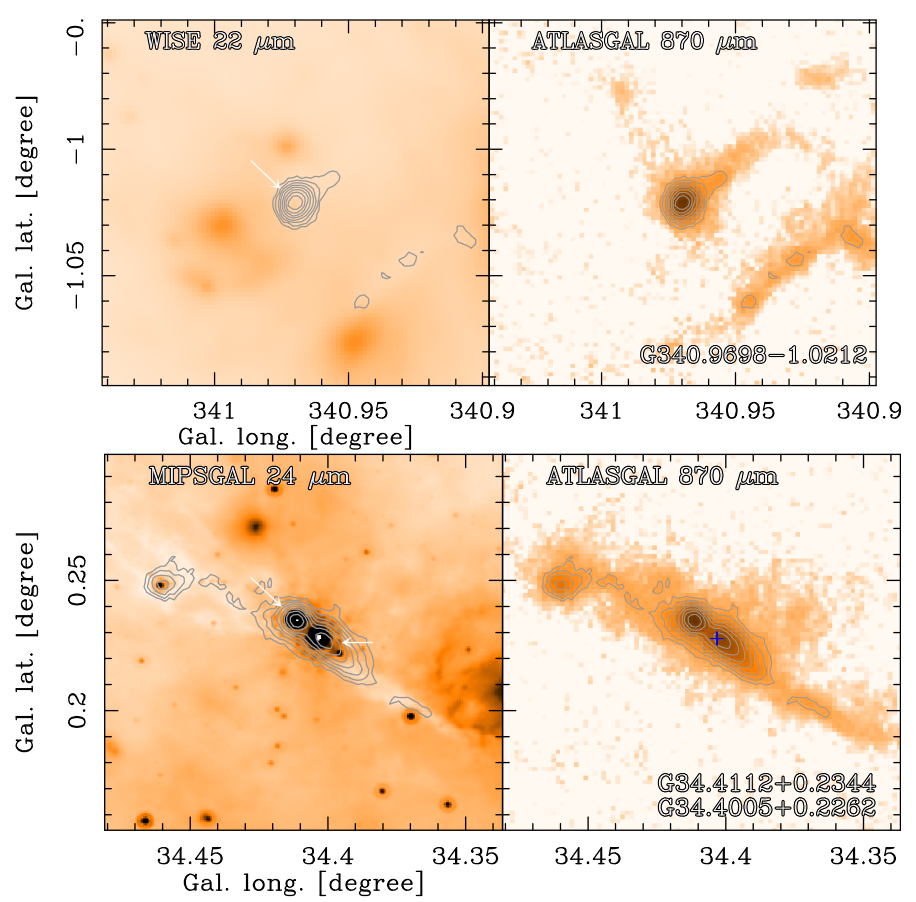

Fig. 2. Top: example of an infrared-quiet massive clump with a single dust peak. The left panel shows the $22-24 \mu \mathrm{m}$ image from WISE/MIPSGAL with contours from ATLASGAL. The white arrows mark the position of infrared-quiet ATLASGAL clumps (Sect. 2.6). The right panel shows the $870 \mu \mathrm{m}$ dust emission from ATLASGAL with the same contours as in the left panel. Bottom: example of a massive clump towards IRDC $34.43+00.24$ (Garay et al. 2004) with a positional shift between the dust and the $24 \mu \mathrm{m}$ point source in the MIPSGAL image, which also coincides with a UC-H II region from CORNISH marked by a blue cross.

Urquhart et al. (2007) to search for ionising emission from stars in our sample of types later than B0.5. Since there is no homogeneous high angular-resolution radio dataset available covering the entire sample towards the IVth Galactic quadrant, we estimate a contamination level by performing the same analysis as above for a larger sample of CORNISH sources.

In Fig. 4 we show the same plot of distance scaled photometry at $22 \mu \mathrm{m}$ versus mass as in Fig. 3 (see Sect. 2.4), but for a larger sample of ATLASGAL sources hosting embedded UC-H II regions from Urquhart et al. (2013b), as well as MYSOs from the RMS survey (Lumsden et al. 2013). This sample has been studied in multiwavelength follow-up observations (for a summary, see Lumsden et al. 2013), and the sources have been classified according whether they host (M)YSOs, $\mathrm{H}_{\text {II }}$ regions, or a mixture of both. We extracted the $22 \mu$ m fluxes for these samples from the WISE catalogue considering matches between the ATLASGAL dust peaks and mid-infrared point sources with a small angular separation $\left(<6^{\prime \prime}\right)$. We considered only good quality flag photometry and a high signal-to-noise ratio of $>10$ in the $22 \mu \mathrm{m}$ band. More importantly, for the RMS and CORNISH sources we only show those with the most reliable distance estimates based on maser parallax measurements (Reid et al. 2014).

We find that massive clumps $\left(M_{\text {clump }}>650 M_{\odot}\right)$ hosting embedded UC-H II regions occupy a well-defined region, and mostly lie above our threshold of $S_{22} \mu \mathrm{m}>289 \mathrm{Jy}$ at $1 \mathrm{kpc}$. Out of the 78 sources of the shown ATLASGAL-CORNISH matches, 5 lie below this threshold, corresponding to $6.4 \%$ of the entire sample. Such weak, compact radio continuum emission has been found towards low bolometric luminosity $\left(L_{\mathrm{bol}} \leq 5 \times 10^{4} L_{\odot}\right)$

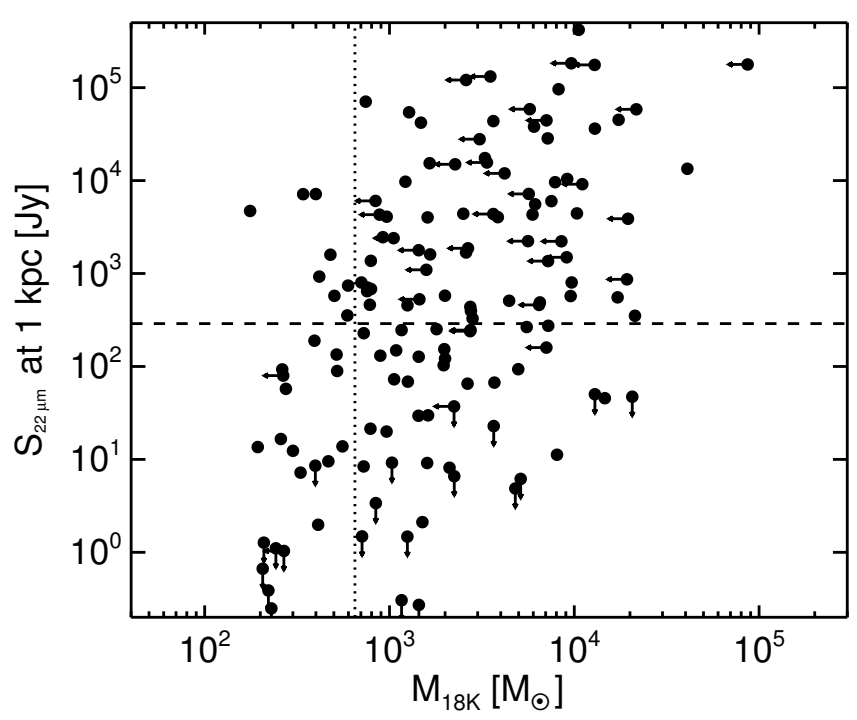

Fig. 3. Distance scaled $22 \mu \mathrm{m}$ flux density based on Spitzer/MIPS photometry and the WISE point source catalogue versus mass estimated from the ATLASGAL flux density. The mid-infrared flux densities have been scaled to a common wavelength. Symbols with arrows pointing left are sources with embedded UC-H II regions and upper limits for their mass estimate. Symbols with arrows pointing down correspond to upper limits for the $22 \mu \mathrm{m}$ photometry. The dashed line shows the 289 Jy flux density threshold discussed in Sect. 2.6, and the dotted line corresponds to $650 M_{\odot}$.

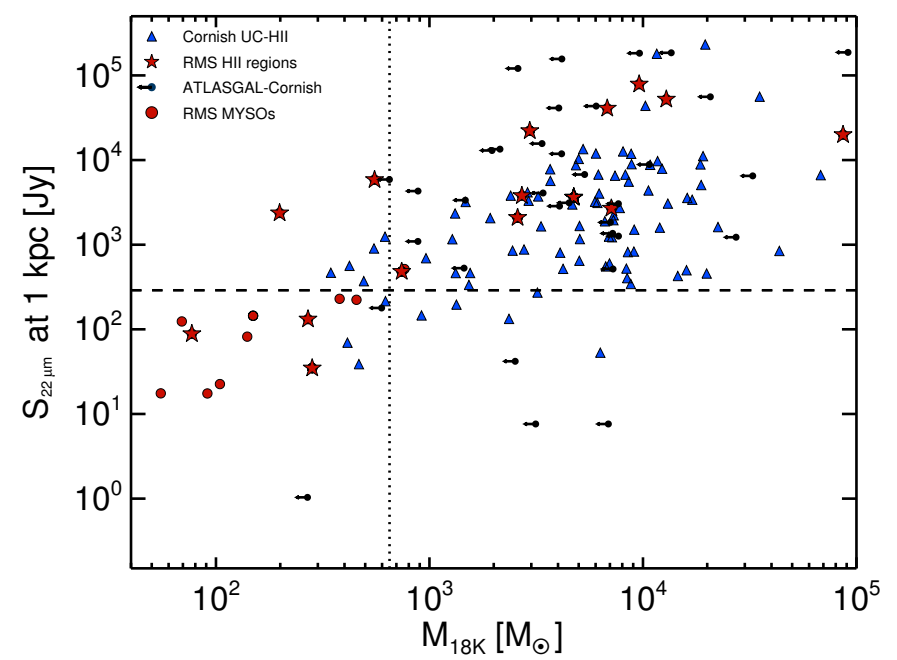

Fig. 4. Mass versus $22 \mu \mathrm{m}$ flux density of the sources covered by the CORNISH survey normalised to a distance of $1 \mathrm{kpc}$. Black filled circles show our sample with a left arrow indicating upper limits for the masses.

MYSOs (see e.g. Guzmán et al. 2010; Lumsden et al. 2013; Avison et al. 2015). They are likely associated with shockionisation from an early outflow activity (e.g. Hoare \& Franco 2007).

Our flux limited selection of ATLASGAL sources with embedded UC-H II regions are also shown as a comparison, and find that 3 sources out of the 31 fall below our mid-infrared threshold, which is $9.7 \%$. We inspected these sources visually, and find that one source lies in a confused region where either the midinfrared photometry may be affected by extended emission, or the radio continuum emission may be associated with the extended $\mathrm{H}$ in region rather than the dust peak. The rest could correspond to the above discussed genuine low bolometric luminosity radio sources associated with MYSOs. To summarise, the 
lowest luminosity massive clumps likely lack embedded UC-H II regions and bright MYSOs, and their contamination below our luminosity threshold is estimated to be less than ten per cent.

\subsection{Classification of the sample}

Using the information described in the previous sections, we classify our sample primarily based on their mid-infrared flux density and indicate the presence of $\mathrm{UC}-\mathrm{H}$ in regions if radio continuum data is available. We do not rely on radio continuum data, however, because sensitive unbiased surveys are not yet available in the IVth quadrant, and thus the sample of UC-H II regions associated with our clumps is incomplete. We introduced the threshold of $289 \mathrm{Jy}$ at $22 \mu \mathrm{m}$ at $1 \mathrm{kpc}$ as discussed in Sect. 2.4, and as empirically justified in Sect. 2.5. Sources above and below this threshold are termed "infrared bright" and "infrared quiet", respectively. This classification is consistent, but simplified compared to previous works (e.g. Csengeri et al. 2014; Giannetti et al. 2014; Csengeri et al. 2016a). In addition, it quantitatively selects the young clumps based on the luminosity of the embedded protostar(s). Contamination of more evolved objects is estimated to be less than $10 \%$ (Sect. 2.5). Nevertheless, in the following we indicate the subsample of massive clumps hosting genuine UC-H II regions for the infrared-bright clumps.

From the sample of 210 sources we were able to classify 209 sources: $139(66 \%)$ are infrared-bright including 51 (24\%) unambiguously associated with UC-H II regions, and 80 $(34 \%)$ are infrared quiet. Considering associations with infrared sources only from the WISE catalogue, we find 56 sources $(26 \%)$ with no counterpart; the remaining $74 \%$ are associated with a mid-infrared source, which is consistent with earlier findings from Csengeri et al. (2014). Some objects are located in regions with complex extended emission at mid-infrared wavelengths, which may affect the photometry, hence their nature being quiet or bright may remain somewhat uncertain. As shown before, this definition of mid-infrared-quiet clumps picks up massive clumps hosting high-mass protostars or their precursors; where no ancillary radio data is available, the contamination from $8-15 M_{\odot}$ main sequence stars is minor. We will investigate the nature of these sources further in Sect. 4. We show the images of infraredquiet sources in Appendix B.

\subsection{SiO (8-7) emission towards infrared-quiet massive clumps}

To further investigate the properties of the infrared-quiet clumps (see also Sect. 4.1), we used spectroscopic observations of a typical shock tracer, $\mathrm{SiO}$, as an indirect probe of ongoing accretion processes. Of the 80 infrared-quiet clumps, we selected 46 objects to follow up with the $\mathrm{FLASH}^{+}$receiver (Klein et al. 2014) at the APEX telescope (Güsten et al. 2006). The source selection was driven by the distance distribution of the sample to favour close-by objects $(d \leq 4.5 \mathrm{kpc})$, and focused on the newly discovered objects (see Sect. 4.2).

Using the APEX/FLASH ${ }^{+}$receiver we covered $8 \mathrm{GHz}$ bandwidth simultaneously centred at $347.15 \mathrm{GHz}$ in USB setup, from which we exploit here the $\mathrm{SiO}(8-7)$ transition at $347.33058 \mathrm{GHz}$. The observations were carried out in 2013 and 2014 August, and we obtained 90" $\times 90^{\prime \prime}$ OTF maps. The reference position was offset by $10^{\prime}$ in right ascension and declination. Pointing and focus were regularly checked during the observations.

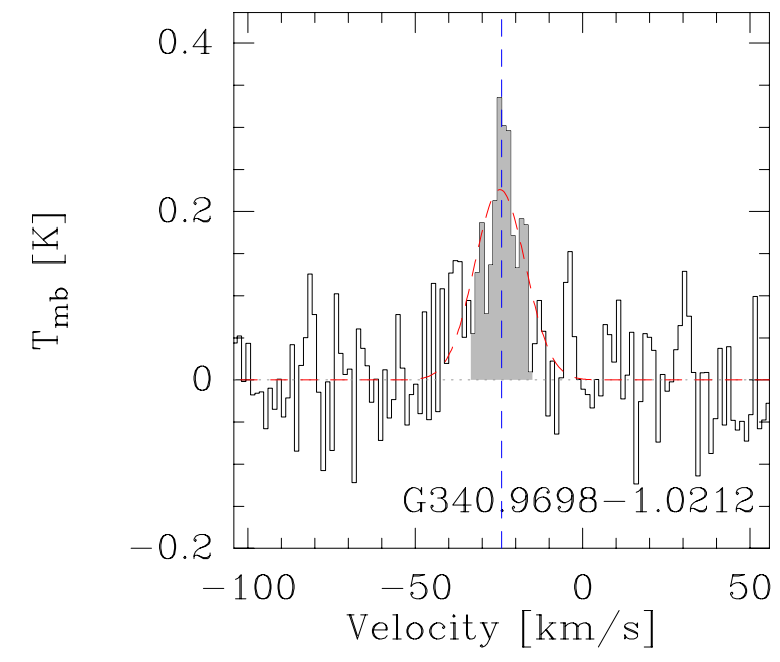

Fig. 5. $\mathrm{SiO}(8-7)$ line observed towards the infrared-quiet massive clump G340.9698-1.0212. The dashed line shows the rest velocity of the source from the $\mathrm{C}^{17} \mathrm{O}(3-2)$ observations at $337.06110 \mathrm{GHz}$ from the same dataset.

The data have been reduced using the GILDAS software following standard procedures. The spectra at the centre, i.e. the dust peak position, have been extracted from the maps. Baselines of order zero have been subtracted and the data have been scaled to the $T_{\mathrm{mb}}$ temperature using $73 \%$ efficiency ${ }^{4}$. The average noise level is $48 \mathrm{mK}$ in $1.3 \mathrm{~km} \mathrm{~s}^{-1}$ resolution.

A total of 27 out of the 46 objects (59\%) show emission of this line above $4 \sigma$ as measured on the line area with Gaussian fitting. We show an example in Fig. 5, while all the detections are shown in Appendix C, Fig. C.1. The observed detection rate is similar to that of Klaassen et al. (2012) despite the lower sensitivity, and is somewhat lower than reported by Leurini et al. (2014), who had, on the other hand, a factor of $\sim 2$ higher sensitivity for the same transition. These studies focused on more evolved, mostly infrared-bright clumps, many of them hosting UC-H II regions. The fact that the high detection rates are broadly similar towards the less luminous thus likely younger clumps is consistent with the findings of Csengeri et al. (2016a) using lower- $J \mathrm{SiO}$ lines.

\section{Statistical properties of the brightest submillimetre sources}

In the following we investigate the distribution and the statistics of the estimated physical properties of the entire sample. As defined in Sect. 2.6 we distinguish between infrared-quiet massive clumps and those classified as infrared-bright, and also show the subsample hosting MYSOs and/or UC-H II regions. Their average physical properties are summarised in Table 2.

\subsection{Mass-distance distribution and completeness}

In Fig. 6 we show the distance distribution of the sample. As discussed in Sect.2.1, a substantial fraction of the sources are part of active star-forming complexes. Their typical distance is around 3-4 kpc, close to the average distance of $4.5 \mathrm{kpc}$ for ATLASGAL clumps (Wienen et al. 2012). Overall $60 \%$ of the sample is located within $4.5 \mathrm{kpc}$; a total of 86 sources are located at greater distances, of which 29 are infrared quiet. The

\footnotetext{
4 http://WWW.apex-telescope.org/telescope/efficiency/
} 


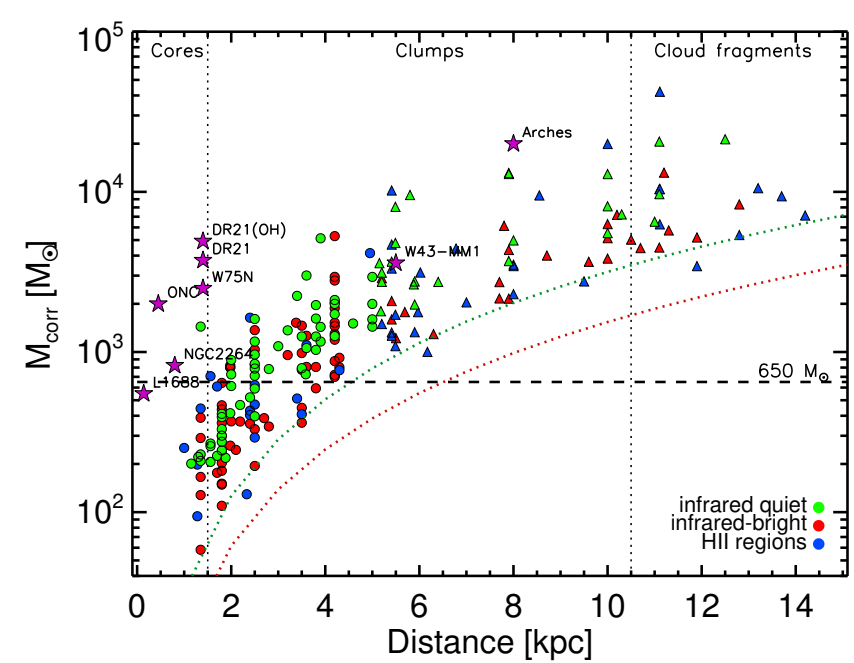

Fig. 6. Temperature corrected mass versus distance distribution. Dotted lines show the mass sensitivity corresponding to the flux density threshold of $5 \mathrm{Jy}$ using $T_{\mathrm{d}}=30 \mathrm{~K}$ for infrared-bright (red), and $T_{\mathrm{d}}=18 \mathrm{~K}$ for infrared-quiet clumps (green). Coloured symbols are shown in the figure legend; filled circles correspond to sources where our selection is complete, while filled triangles show sources where the sample is incomplete above $650 M_{\odot}$. The dashed horizontal line shows the $650 M_{\odot}$ limit for massive clumps potentially capable of forming high-mass stars from Csengeri et al. (2014). Known (proto)clusters from the literature are labelled with magenta stars, as are the estimated initial clump mass at half-radius for the galactic (super) star clusters like the Orion Nebula Cluster (ONC, e.g. Da Rio et al. 2012) and Arches (Habibi et al. 2013), following Tan et al. (2014). The approximate distance limit for detecting sources corresponding to cores, clumps, and cloud fragments is shown as a dotted line (Bergin \& Tafalla 2007).

more distant sources are therefore dominated by infrared-bright clumps. We also show a threshold of $650 M_{\odot}$, introduced in Csengeri et al. (2014), which is an extrapolation of the massive dense core definition by Motte et al. (2007) to the $\sim 0.3 \mathrm{pc}$ scale of ATLASGAL clumps. Empirically this selection has proved to select cores hosting high-mass protostars by Bontemps et al. (2010).

The mass estimation corresponding to the threshold of $5 \mathrm{Jy}$ used for our flux limit is indicated in Fig. 6 as green and red dotted lines for infrared-bright $\left(T_{\mathrm{d}}=30 \mathrm{~K}\right)$ and infrared-quiet clumps $\left(T_{\mathrm{d}}=18 \mathrm{~K}\right)$, respectively. It shows that our selection is complete for infrared-quiet clumps above $650 M_{\odot}$ up to a distance of $4.5 \mathrm{kpc}$, while for infrared-bright clumps above $310 M_{\odot}$. Since we expect the more evolved clumps to have lost a part of their mass in the central region through cluster formation and dissipation, we can argue that this sample is roughly complete for all clumps having $650 M_{\odot}$ at birth at all their stages of evolution from infrared quiet to infrared bright and possibly also UC$\mathrm{H}_{\text {II }}$ regions. A total of 28 infrared-quiet and 55 infrared-bright clumps are found above $650 M_{\odot}$ and $310 M_{\odot}$ at a distance closer than $4.5 \mathrm{kpc}$.

The area covered by the sector of our Galaxy from $\ell=-60^{\circ}$ to $60^{\circ}$ and for a distance lower than $4.5 \mathrm{kpc}$ is found to represent $\sim 10 \%$ of the total area of the galactic disk within the solar circle after removing the inner $3 \mathrm{kpc}$. In a rough approximation, we can consider this $10 \%$ as the fraction of star formation in the Galactic disk which is occurring in our distance limited sample.

\subsection{Temperature distribution}

From the $\mathrm{NH}_{3}$ data discussed in Sect. 2.2 we determine a median kinetic temperature of $25.5 \mathrm{~K}$ for $60 \%$ of the sample. Figure 7

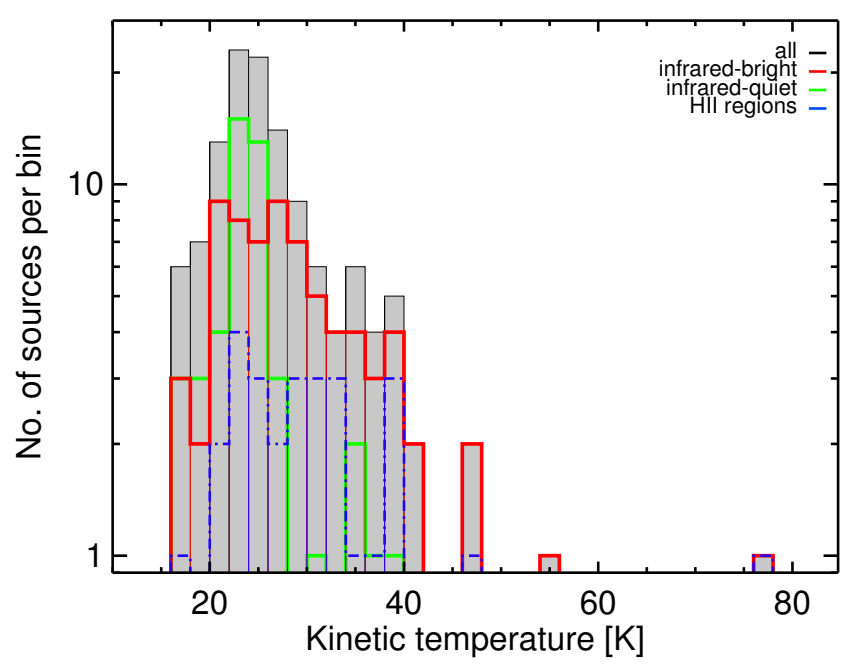

Fig. 7. Distribution of kinetic temperature from $\mathrm{NH}_{3}(1,1)$ and $(2$, 2) measurements from Wienen et al. (2012) and (2017). The infraredbright and infrared-quiet sources are shown in different colours, as explained in the figure legend. We also show massive clumps hosting UC-H II regions as a subsample of the infrared-bright sources.

shows the temperature distribution of these sources. In this sample 28 have a firm association with UC-H II-regions, and the majority of the sources are classified as infrared bright, in total 71 . These types of sources have a median kinetic temperature of 29.6 and $27.2 \mathrm{~K}$, with a standard deviation of $8-11 \mathrm{~K}$. Towards infrared-quiet sources we find a median of $23.8 \mathrm{~K}$ with a standard deviation of $4.7 \mathrm{~K}$. This suggests a trend of lower temperatures towards younger sources; however, given their large standard deviation, this difference is not statistically significant. Following our argumentation in Sect. 2.4, and considering the similar temperatures, here we do not make a distinction between massive clumps with UC-H II regions and those hosting infrared-bright sources. A more accurate temperature assignment to the group of infrared-quiet massive clumps still remains uncertain since the sources with available $\mathrm{NH}_{3}$ measurements are dominated by the more evolved sources, and the targeted infrared-quiet clumps may not be representative of the entire subsample.

\subsection{Distribution of physical properties}

In Fig. 8 we show the mass distribution of the sample, where we adopted $T_{\mathrm{d}}=30 \mathrm{~K}$ for the infrared-bright sources and kept $T_{\mathrm{d}}=18 \mathrm{~K}$ for the infrared-quiet clumps (see Sect. 2.3). We give an overview of the statistical properties of the physical parameters of all sources and the two source types in Table 2. The global statistical properties of the infrared-bright and -quiet samples are similar, and we find a remarkably flat mass distribution for all source types, the majority of the sources lying between $650 M_{\odot}$ and $8000 M_{\odot}$. Lower mass objects correspond to nearby massive dense cores with masses between $\sim 86-120 M_{\odot}$. Towards sources at greater distances we find masses of $\sim 10^{5} M_{\odot}$ corresponding to cloud fragments rather than clumps. The mean clump mass is $\sim 4.5 \times 10^{3} M_{\odot}$, which is about a factor of two higher compared to the star formation selected larger sample of ATLASGAL clumps studied by Urquhart et al. (2014).

The size distribution of the sample is shown in Fig. 9, and as above, there is no significant difference between the different source types. Adopting the nomenclature of Williams et al. (2000, see also Bergin \& Tafalla 2007), we find a small fraction of sources that correspond to cores or entire cloud fragments, 
T. Csengeri et al.: The ATLASGAL survey: The sample of young massive cluster progenitors

Table 2. Statistics of the physical properties of all sources, and the infrared-bright and infrared-quiet samples.

\begin{tabular}{|c|c|c|c|c|c|}
\hline & \multicolumn{5}{|c|}{ All sources } \\
\hline & Min & Max & Mean & Median & Stdev \\
\hline Distance $[\mathrm{kpc}]$ & 1.00 & 15.6 & 4.9 & 3.9 & 3.30 \\
\hline$R_{90}[\mathrm{pc}]$ & 0.07 & 1.63 & 0.40 & 0.32 & 0.28 \\
\hline$M_{18 \mathrm{~K}}\left[M_{\odot}\right]$ & 120 & $0.9 \times 10^{5}$ & $4.5 \times 10^{3}$ & $1.8 \times 10^{3}$ & $7.9 \times 10^{3}$ \\
\hline$M_{\text {corr }}\left[M_{\odot}\right]$ & 58 & $4.2 \times 10^{5}$ & $2.8 \times 10^{3}$ & $1.3 \times 10^{3}$ & $4.6 \times 10^{3}$ \\
\hline$\Sigma_{\text {corr }}\left[\mathrm{g} \mathrm{cm}^{-2}\right]$ & 0.27 & 5.9 & 1.11 & 0.75 & 1.02 \\
\hline \multirow[t]{2}{*}{$\bar{n}_{\text {corr }}\left[\times 10^{5} \mathrm{~cm}^{-3}\right]$} & 0.14 & 44.15 & 3.17 & 1.56 & 5.25 \\
\hline & \multicolumn{5}{|c|}{ Infrared-bright massive clumps } \\
\hline Distance $[\mathrm{kpc}]$ & 1.0 & 14.20 & 5.14 & 4.20 & 3.43 \\
\hline$R_{90}$ & 0.07 & 1.41 & 0.42 & 0.35 & 0.27 \\
\hline $\operatorname{Mass}_{18 \mathrm{~K}}\left[M_{\odot}\right]$ & 120 & $8.7 \times 10^{4}$ & $5.2 \times 10^{3}$ & $2.4 \times 10^{3}$ & $9.3 \times 10^{3}$ \\
\hline Mass $_{\text {corr }}\left[M_{\odot}\right]$ & 58 & $4.2 \times 10^{4}$ & $2.7 \times 10^{3}$ & $1.2 \times 10^{3}$ & $4.7 \times 10^{3}$ \\
\hline$\Sigma_{18 \mathrm{~K}}\left[\mathrm{~g} \mathrm{~cm}^{-2}\right]$ & 0.27 & 6.00 & 0.99 & 0.56 & 1.14 \\
\hline \multirow[t]{2}{*}{$\bar{n}_{18 \mathrm{~K}}\left[\times 10^{5} \mathrm{~cm}^{-3}\right]$} & 0.14 & 44.15 & 2.78 & 1.03 & 5.89 \\
\hline & \multicolumn{5}{|c|}{ Infrared-quiet massive clumps } \\
\hline Distance $[\mathrm{kpc}]$ & 1.15 & 15.60 & 4.47 & 3.70 & 3.05 \\
\hline$R_{90}$ & 0.08 & 1.63 & 0.36 & 0.28 & 0.28 \\
\hline $\operatorname{Mass}_{18 \mathrm{~K}}\left[M_{\odot}\right]$ & 200 & $2.1 \times 10^{4}$ & $3.1 \times 10^{3}$ & $1.4 \times 10^{3}$ & $4.5 \times 10^{3}$ \\
\hline$\Sigma_{18 \mathrm{~K}}\left[\mathrm{~g} \mathrm{~cm}^{-2}\right]$ & 0.51 & 4.05 & 1.31 & 1.03 & 0.74 \\
\hline $\bar{n}_{18 \mathrm{~K}}\left[\times 10^{5} \mathrm{~cm}^{-3}\right]$ & 0.20 & 18.46 & 3.83 & 2.39 & 3.91 \\
\hline
\end{tabular}

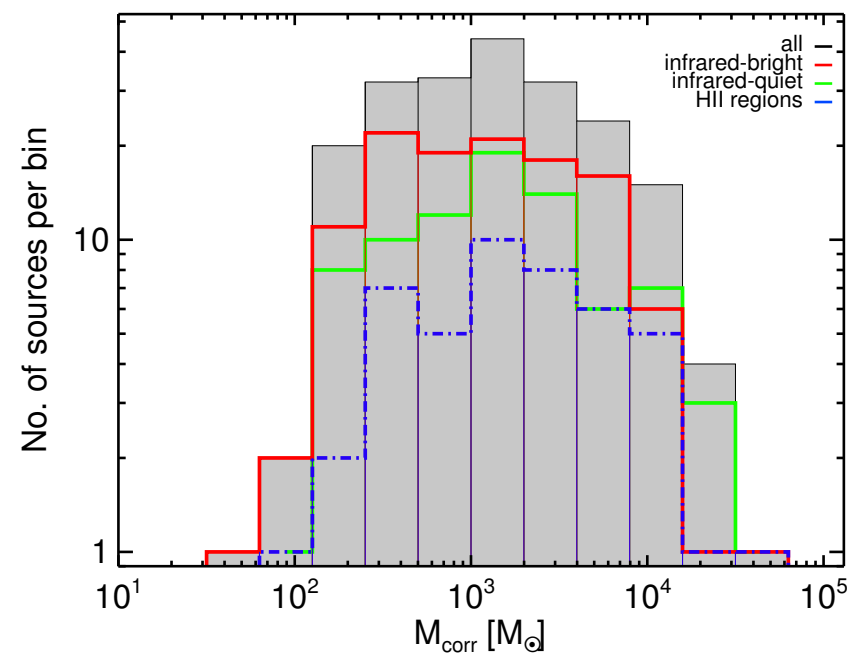

Fig. 8. Temperature corrected mass distribution (using $T_{\mathrm{d}}=30 \mathrm{~K}$ for the infrared-bright clumps). Colours are the same as in Fig. 7. The clumps hosting genuine UC-H II regions are a subsample of the infrared-bright clumps.

while the majority of the sources correspond to clumps. They have typical sizes of $0.3-0.4 \mathrm{pc}$, which is comparable to the mean size of $\sim 0.36 \mathrm{pc}$ found by Russeil et al. (2010) in NGC 6334/NGC 6357. Compared to the population of massive dense cores in Cygnus- $X$, the sizes are $\sim 3$ times larger, while the masses are on average $\sim 5$ times larger, suggesting their potential to form high-mass stars.

In Fig. 10 we show the mass surface density distribution of the sample. Lower surface densities are found towards the infrared-bright sources, a considerable fraction of which host UC-H II regions. This is likely due to an observational bias, driven both by a larger fraction of such sources at a larger distance, which then correspond to larger structures with lower average surface density, and the lower mass selection threshold for

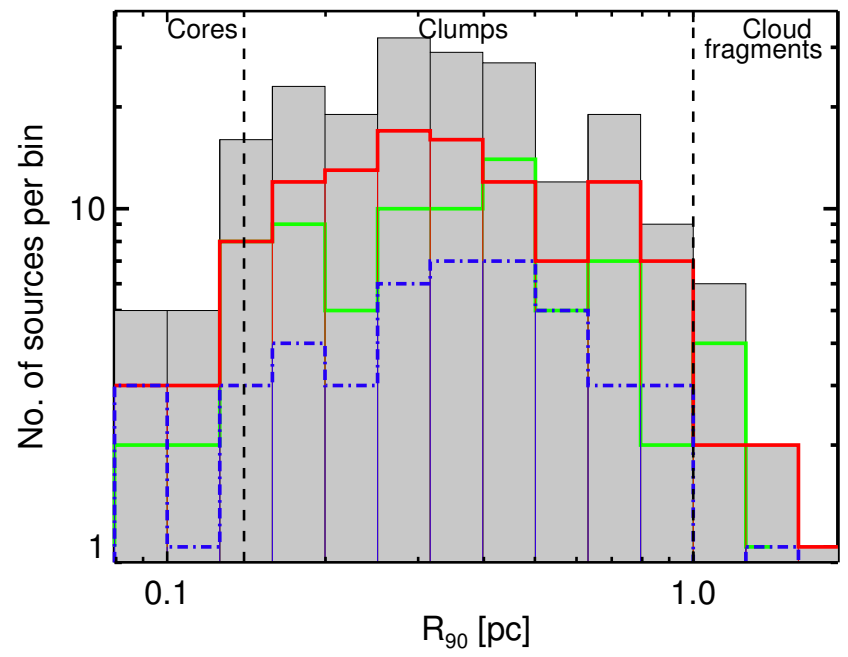

Fig. 9. Distribution of the deconvolved physical size $\left(R_{90}\right)$ of the sample. Colours are the same as in Fig. 7. The dashed lines indicate an approximate distinction between cores, clumps, and cloud fragments (Bergin \& Tafalla 2007).

the infrared-bright sources. The mean surface density is above the theoretical value of $\Sigma_{\mathrm{cl}} \geq 1 \mathrm{~g} \mathrm{~cm}^{-2}$ by Krumholz \& McKee (2008), suggesting that a substantial fraction of our sources have the potential to form high-mass stars. Correspondingly, the median volume-averaged density of $3.13 \times 10^{5} \mathrm{~cm}^{-3}$ is higher than that found in other samples in the literature (e.g. Russeil et al. 2010), and suggests that we probe here high density, starforming gas.

\section{Nature of the brightest submillimetre sources in the inner Galaxy}

The brightest submillimetre sources in the inner Galaxy are generally associated with active sites of star formation. Here we 


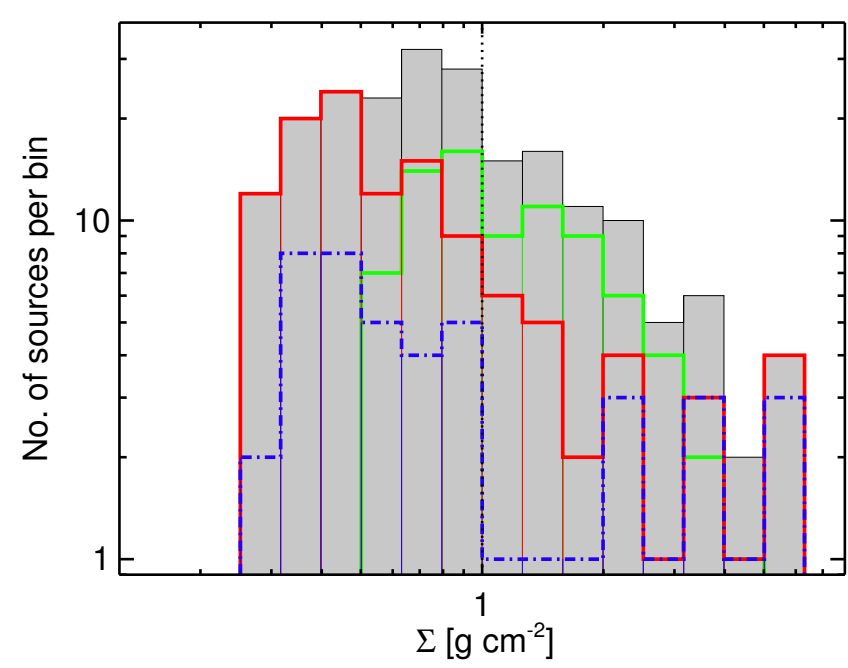

Fig. 10. Mass surface density $(\Sigma)$ distribution of the sample using $T_{\mathrm{d}}=$ $30 \mathrm{~K}$ for the infrared-bright clumps. Colours and symbols are the same as in Fig. 7.

first investigate the star formation potential and evolutionary aspect of the entire sample (Sect.4.1), and argue that the majority of the infrared-quiet massive clumps are potential precursors of massive stars and/or clusters. We then show that our sample of infrared-quiet clumps contains sources previously not recognised in other surveys using infrared emission, or absorption (Sect. 4.2). Finally, we search for signposts of embedded protostars in the infrared-quiet subsample to assess the stage of ongoing star formation (Sect.4.3). We then discuss the stability of the entire sample (Sect.4.4), and provide predictions for their small-scale fragmentation (Sect. 4.5).

\subsection{Precursors of massive clusters}

Comparing the physical properties in terms of mass, size, and density, in Table 2 we show that our sample exhibits rather homogeneous properties, and no statistically significant differences are found between the two source types. This includes well-studied Galactic mini-starburst regions, for instance the W43 ridge (e.g. Motte et al. 2003; Bally et al. 2010; Nguyen Luong et al. 2011), W51 Main (e.g. Mufson \& Liszt 1979; Zapata et al. 2008; Ginsburg et al. 2015), and W49N (e.g. Peng et al. 2010; Galván-Madrid et al. 2013). While indications of ongoing star formation is prevalent in the sample, are all clumps prone to form mini-starbursts and rich clusters? We compare our results with the empirical relation of Kauffmann \& Pillai (2010) in Fig. 11, which suggests that all of our sources have the potential to form high-mass stars. While the comparative statistical analysis of infrared-quiet and -bright sources reveals similar physical characteristics in terms of mass and size, they seem to differ only in their embedded (proto-)stellar population. Low bolometric luminosity sources with similar physical characteristics to their more evolved counterparts have been frequently interpreted as being in an earlier evolutionary stage (e.g. Motte et al. 2007; Molinari et al. 2008). This suggests that infrared-quiet clumps are the precursors of the infrared-bright clumps and UC-H II regions, and the sample can be arranged along a similar evolutionary path.

The large mass reservoir of infrared-bright sources suggests that they likely continue producing high-mass stars. Unless specific conditions are required for the formation of rich clusters, we shall have selected here their true precursors.

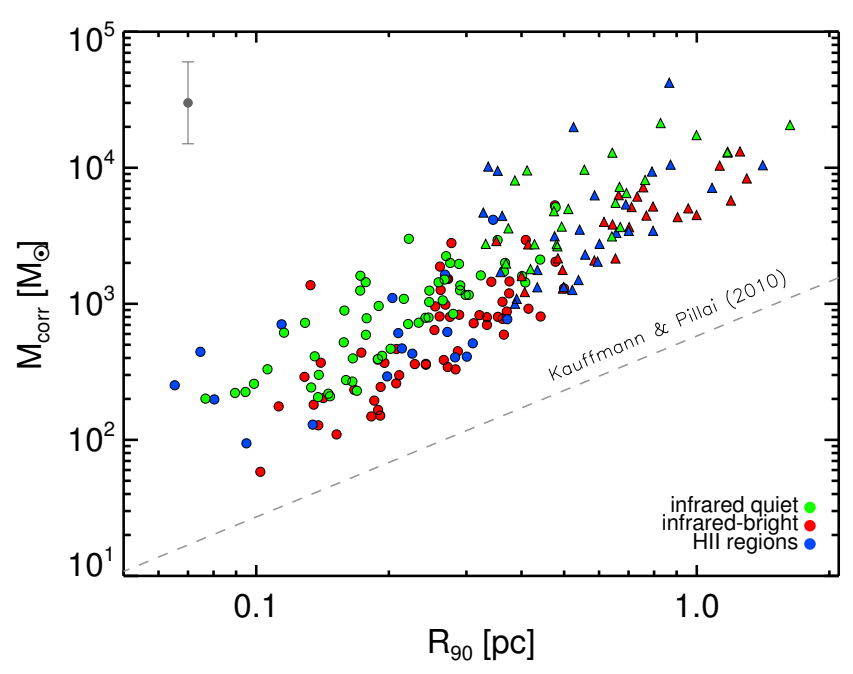

Fig. 11. Temperature corrected mass estimates (using $T_{\mathrm{d}}=30 \mathrm{~K}$ for the infrared-bright clumps) versus deconvolved physical size $\left(R_{90}\right)$ from Sect. 2.3. Dashed lines show the relation of Kauffmann et al. (2010) scaled to the Ossenkopf \& Henning (1994) dust opacity following Dunham et al. (2011). In the upper left corner we show the factor of two uncertainty in the mass estimate. Colours are the same as in Fig. 7.

\subsection{Massive cluster progenitors in isolation?}

Because the clustered nature of high-mass star formation was recognised early on, several studies searched for younger objects in the vicinity of bright, luminous sources (e.g. Garay et al. 2004; Thompson et al. 2005). To look for neighbouring, more evolved objects with UC-H II regions in the close vicinity of the infrared-quiet sample, we used the CS (2-1) survey of IRAS sources (Bronfman et al. 1996), which is complete towards IRAS selected UC-H II regions in the Galactic plane. In total we found 92 associations (42\%) within $0.8^{\prime}$ offset, which corresponds to $0.26 \mathrm{pc}$ for the nearest source and $2.9 \mathrm{pc}$ for the most distant source in our sample. However, in the infrared-quiet sample only $29(36 \%)$ are found in the vicinity of an IRAS source, and their offsets correspond to a physical separation between 0.3-16.3 pc. Compared with the more sensitive and higher angular resolution Red MSX survey (RMS, Lumsden et al. 2013), we find six infrared-quiet clumps in the vicinity of a bright MYSO with $L_{\text {bol }}>2 \times 10^{4} L_{\odot}$.

Surveys sensitive to cold dust are better suited to directly probe the youngest clumps. To evaluate the fraction of previously identified infrared-quiet clumps, we first looked for associations with cloud structures identified as infrared-dark clouds (IRDCs) by correlating the peak dust positions with the catalogues of IRDCs revealed by MSX (Jackson et al. 2008; Simon et al. 2006) and Spitzer (Peretto \& Fuller 2009). In total we find 20 associations with an MSX Dark Cloud within 120", 15 of which are infrared-quiet sources. For the association with Spitzer Dark Clouds we used the Herschel-confirmed clouds from Peretto et al. (2016) with a smaller positional offset of 50" corresponding to their average equivalent size of $\sim 25^{\prime \prime}+1 \sigma$, where $\sigma$ is their standard deviation. In total, 65 of our clumps have associations, 31 of which are infrared-quiet clumps. This means that $\sim 61 \%$ of the infrared-quiet clumps have not been recognised before in these surveys.

Thus, we find that the infrared-quiet sample is found in relative isolation compared to the bright clumps which already undergo active star formation. Less than half of the ATLASGAL selected massive clumps have IRDC counterparts, and comparison with the star formation selected ATLASGAL clumps of 
Urquhart et al. (2014) shows that 25 of the infrared-quiet sources are newly identified. In summary, we find that $\sim 23 \%$ of our infrared-quiet sample has not been recognised by these previous studies.

\subsection{Signposts of already formed embedded high-mass protostars}

To tackle the stage of ongoing star formation, here we compare our sample with tracers of high-mass star formation. We found a total of 23 clumps coinciding with extended green objects (EGOs); (Cyganowski et al. 2008; and Chen et al. 2013) most of them are infrared-quiet clumps, 2 sources are infrared-bright, and 3 are UC-H II regions. Since EGOs are believed to trace powerful outflows mostly from (presumably massive) Class 0 YSOs, it confirms that the infrared-quiet clumps host the youngest protostellar phases. However, only $25 \%$ of the infrared-quiet clumps are associated with EGOs. This may indicate that the EGO phase is shorter than the infrared-quiet clump phase, and therefore does not represent well the entire early evolutionary phase of massive clumps. The few EGOs associated with infrared-bright clumps may indicate that young massive protostars can still form at the time of the infrared-bright phase, and even perhaps at the UC$\mathrm{H}$ II phase if star formation is a continuous process in clumps.

On the other hand, the lack of EGOs does not necessarily imply a lack of outflow activity, since high extinction, even at $4.5 \mu \mathrm{m}$, may prevent their detection. In our $\mathrm{SiO}(8-7)$ spectroscopic study, $\sim 60 \%$ of infrared-quiet clumps show emission, which is a much larger fraction than that of EGOs. Using the lower energy $\mathrm{SiO}$ (2-1) line, Csengeri et al. (2016a) find that an even higher fraction of the infrared-quiet massive clumps exhibit emission in this shocked gas tracer. This is consistent with a picture that infall and accretion processes may already be occurring in at least some of the infrared-quiet clumps, indirectly pointing at an underlying population of deeply embedded protostars (see the example of NGC 6334 I(N) by Megeath \& Tieftrunk 1999).

Another tracer of the nature of embedded protostars is Class II methanol maser emission. These masers are excited by radiative pumping requiring the intense mid-infrared emission from the warm, dense material of deeply embedded hot-cores or UC-H II regions (Menten 1991; Sobolev et al. 1997). We therefore cross-correlated our sample with the Methanol MultiBeam survey (Caswell et al. 2010, 2011; Green et al. 2010; Breen et al. 2015), and found $138(66 \%)$ associations within 20" , of which 57 are infrared-quiet massive clumps corresponding to $\sim 71 \%$ of the subsample. Methanol masers thus seem to trace a larger fraction of the infrared-quiet phase, although less selectively since they are also found towards the more evolved stages of clump evolution. Altogether we find that a considerable fraction of the infrared-quiet sample show signs of embedded (high-mass) protostars and ongoing star formation process.

\subsection{Stability estimates and timescales}

Using high-density tracers we estimate here the virial mass and compare it to the mass estimates based on the dust emission. As a first step we used the $\mathrm{NH}_{3}(1,1)$ line width measurements of Wienen et al. (2012) and Wienen et al. (2017) where available, and then complemented these data with measurements of the CS (2-1) line from Bronfman et al. (1996), and finally of the $\mathrm{H}^{13} \mathrm{CO}^{+}(1-0)$ line from recent surveys using the Mopra (Wyrowski et al., in prep.) and the IRAM 30 m telescopes (Csengeri et al. 2016a). We have line width estimates for a total

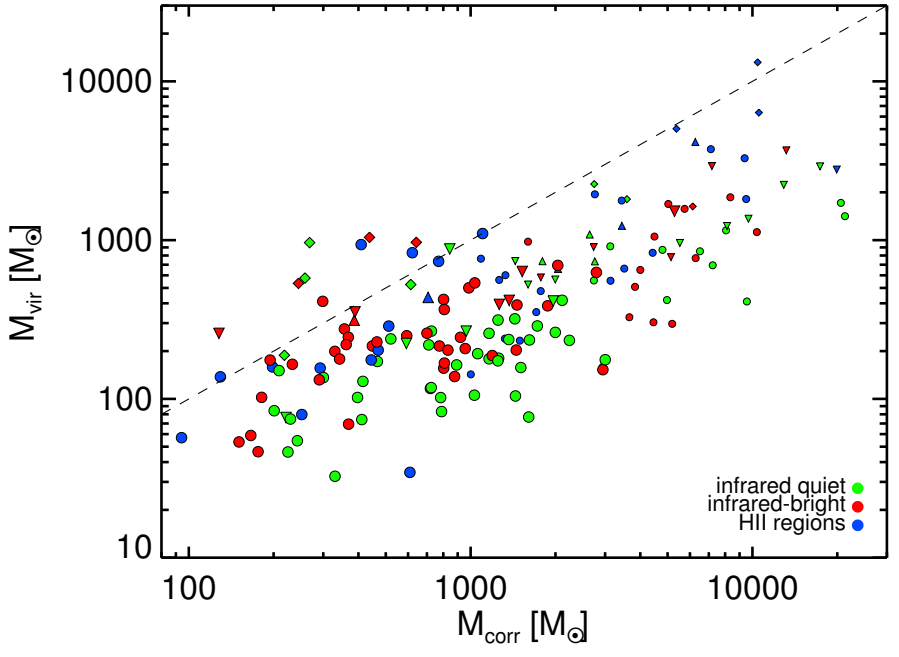

Fig. 12. Temperature corrected clump mass estimates compared to the virial mass. The dashed line indicates where the virial mass equals the dust mass. Colours are the same as in Fig. 7, filled circles correspond to line width estimates from Wienen et al. (2012, 2015), filled upward triangle to that of Csengeri et al. (2016a), filled downward triangle to that of Wyrowski et al. (in prep.), filled diamonds to that of Bronfman et al. (1996). Sources with $d>5 \mathrm{kpc}$ are plotted in smaller symbols.

of 173 sources, $80 \%$ of the whole sample. While this is a smaller sample than studied in Wienen et al. (2012) and Urquhart et al. (2014), it is more focused on the brightest ATLASGAL clumps.

We estimate the virial mass assuming a density profile of $n(r) \sim r^{-2}$, and using $M_{\mathrm{Vir}}=3 \times \frac{R_{90} \times \sigma_{\mathrm{tot}}^{2}}{G}$ (Bertoldi \& McKee 1992), where $R_{90}$ is the radius of a sphere, $\sigma_{\text {tot }}$ is the velocity dispersion of the gas, and $G$ is the gravitational constant,

$\frac{M_{\mathrm{Vir}}}{\left[M_{\odot}\right]}=697 \frac{R_{90}}{[\mathrm{pc}]} \frac{\sigma_{\mathrm{tot}}^{2}}{\left[\mathrm{~km} \mathrm{~s}^{-1}\right]}$.

As shown in Fig. 12, we find surprisingly small $M_{\mathrm{Vir}} / M$ ratios towards the majority of the sources, on average 0.47 with a median of 0.29 , strongly suggesting that they are gravitationally unstable. While the used molecular tracers selectively probe the high-density gas, uncertainties due to the different tracers and beam sizes could affect our virial mass estimates. However, despite the various datasets used, we see no systematics in the estimated virial masses. As Fig. 12 shows, 155 sources (89\%) have a smaller virial mass than their estimated gas mass. We find the same trend for the more distant sources of the sample, which are beyond our completeness limit. Uncertainties in the mass estimates due to the dust opacity and gas-to-dust ratio would lower the $M_{\mathrm{Vir}} / M$ ratio by a factor of two. Even considering the case of a uniform $n(r) \sim r^{0}$ density profile, we would still find a large fraction (67\%) of the clumps having $M_{\mathrm{Vir}} / M<1$, which could thus still be considered as gravitationally unstable. Magnetic fields could, however, slow down the rate of collapse. For a large number of clouds Kauffmann et al. (2013) provides estimates of the virial parameter defined by $M_{\mathrm{Vir}} / M$, and find similarly low values. They suggest that magnetic fields as strong as $1 \mathrm{mG}$ would be required to slow down the collapse, otherwise they undergo rapid collapse.

The size-scale of clumps, $0.2-1 \mathrm{pc}$, is large compared to the scale of MDCs $(<0.15 \mathrm{pc})$ and cores $(<0.05 \mathrm{pc})$ for which collapse signatures are frequently observed. Our results therefore suggest that these massive cluster progenitors are already collapsing on a global scale, i.e. a clump scale. This is particularly intriguing because it suggests that gravity could be the 
Table 3. Comparison of the properties of the infrared-quiet sources with the literature.

\begin{tabular}{lcccc}
\hline \hline & $\begin{array}{c}\text { ATLASGAL infrared-quiet } \\
\text { clumps }\end{array}$ & $\begin{array}{c}\text { Herschel-IRDC } \\
\text { clumps }\end{array}$ & $\begin{array}{c}\text { IRDC } \\
\text { clumps }\end{array}$ & $\begin{array}{c}\text { CygX } \\
\text { clumps }\end{array}$ \\
\hline Distance $[\mathrm{kpc}]$ & $1.15-15.60(4.47)$ & $0.12-16.45(4.18)$ & $1.8-7.1(3.7)^{b}$ & $1.4^{d}$ \\
Size $[\mathrm{pc}]$ & $0.08-1.63(0.36)$ & $0.02-2.6(0.6)$ & $1-3^{b}$ & $0.56^{e}$ \\
$M\left[M_{\odot}\right]^{\ddagger}$ & $200-21000(1400)$ & $0.11-16000(700)$ & $120-16000(950)^{b}$ & $680^{e}$ \\
$\bar{n}_{\text {corr }}\left[\times 10^{5} \mathrm{~cm}^{-3}\right]$ & $0.20-18.46(2.39)$ & $0-0.58(0.01)$ & $0.001-0.1^{b}$ & $0.12^{e}$ \\
\hline
\end{tabular}

Notes. ${ }^{(\dagger)}$ Range of minimum to maximum values, the median is given between parentheses. ${ }^{(\ddagger)}$ For the adopted dust temperature see their original work. ${ }^{(a)}$ Adopted from the values listed in Table 2 from Traficante et al. (2015). ${ }^{(b)}$ Adopted from the values listed in Table 3 from Rathborne et al. (2006). ${ }^{(c)}$ Adopted from the values listed in Table 1 from Rathborne et al. (2006). ${ }^{(d)}$ Distance from Rygl et al. (2012). ${ }^{(e)}$ Scaled to $1.4 \mathrm{kpc}$ from Motte et al. (2007).

dominant force in the mass assembly process over size-scales of entire clumps, which are larger than previously considered (e.g. McKee \& Ostriker 2007).

We also calculate the free-fall time of these clumps by $\tau_{\mathrm{ff}}=\sqrt{3 \pi / 32 G n}$, where $G$ is the gravitational constant, and $n$ is the volume density. We find on average $5.64 \times 10^{4} \mathrm{yr}$, which is somewhat shorter than the lifetime estimate of $7.5 \times 10^{4} \mathrm{yr}$ by Csengeri et al. (2014). Such relative collapse timescales (i.e. a few times the free-fall timescale) are typically observed towards low-mass cores (see Ward-Thompson et al. 2007, and references therein), and have recently been suggested for the formation of high-mass cores by Duarte-Cabral et al. (2013).

Collapse timescales of a few times $10^{4} \mathrm{yr}$ suggests that all massive clumps, precursors of massive clusters, are transient entities and should disappear on short timescales, typically less than $10^{5} \mathrm{yr}$ (see also Zinnecker \& Yorke 2007; Motte et al. 2007). Embedded MYSOs and UC-H II evolve, however, on longer timescales, of the order of $10^{5}-10^{6} \mathrm{yr}$ according to stellar evolution models with accretion rates of $M_{\text {acc }} \sim 10^{-3} M_{\odot} \mathrm{yr}^{-1}$ (e.g. Churchwell 2002; Hoare et al. 2007; Kuiper \& Yorke 2013). This is at least up to an order of magnitude longer than estimated for the collapse of their parent clump. This means that the clump material should have already collapsed leaving a considerably less dense and less massive clump towards the more evolved objects. This apparent contradiction to the observations can only be explained if there is mass replenishment onto the clump on large scales fuelling a continuous star formation, or if we selectively pick here the youngest of the galactic UC-H II regions still surrounded by their natal clump. Case studies of massive cluster progenitors show evidence both for global collapse and for flows of dense gas (e.g. Schneider et al. 2010; Csengeri et al. 2011a,b; Peretto et al. 2013; Tackenberg et al. 2014). This points to a scenario where massive clumps undergo star formation, while gas replenishment arrives on larger scales, and maintains the observed similar mass range for these clumps.

\subsection{Initial stages of massive proto-clusters: prediction for fragmentation properties}

We overview the physical properties of our infrared-quiet sample in comparison with typical IRDCs (Rathborne et al. 2006), Herschel clumps embedded in IRDCs (Traficante et al. 2015), and clumps in the Cygnus-X high-mass star-forming region (Motte et al. 2007) in Table 3. While IRDCs are typically more extended structures, they have lower masses and exhibit smaller volume densities. Herschel selected clumps span a much broader range of physical conditions and cover similarly high-mass clumps as our sample. They are found to be, however, more extended and are therefore on average lower density structures. The ATLASGAL counterparts of IRDCs selected by Kainulainen \& Tan (2013) correspond to sources below the flux density threshold used for this study. The ATLASGAL sources used here are the brightest and therefore the most massive among the compact millimetre sources in the inner Galaxy. Their typical size of $\sim 0.4 \mathrm{pc}$ corresponds to protoclusters. We can therefore discuss our sample in terms of the precursors of the richest clusters in the Galaxy.

In Fig. 13 we show the temperature corrected mass versus the surface density distribution of the entire sample following Tan et al. (2013), with different colours corresponding to the infrared-bright and infrared-quiet subsamples. It is clear that the sample discussed here lies, on average, close to the theoretical limit for high-mass star formation around $\Sigma \simeq 1 \mathrm{~g} \mathrm{~cm}^{-2}$ (Krumholz \& McKee 2008) supporting the scenario that it is capable of forming high-mass stars and clusters. Taking the two extreme profiles of constant density $n(r) \sim r^{0}$ and power law with $n(r) \sim r^{-2}$, we mark with stars the predicted fragmentation on scales smaller by a factor of ten, approaching the size-scales of a few thousand AU corresponding to individual collapsing objects (e.g. Bontemps et al. 2010). The uniform density profile, where the surface density stays constant, predicts fragments below solar mass, which would correspond to clumps forming a rich cluster of low-mass stars. The case of the steeper density profile predicts more massive structures on smaller scales, reaching a mass range of several tens of solar masses. Envelope masses of this order are expected to form high-mass stars.

Some of the well-studied sources of our selection have been subject to high angular resolution follow-ups. The extreme clumps of the W43 complex, MM1 to MM3 (Motte et al. 2003; Sridharan et al. 2014) hosts one of the most extreme sites of active high-mass star formation in terms of mass and youth (Louvet et al. 2014). Massive envelopes have been identified towards other clumps as well, for example G35.03+0.35 (G35.0250+0.3501) (Beltrán et al. 2014), G35.200.74 (G35.1976-0.7427) (Sánchez-Monge et al. 2013), and SDC335-MM1(G335.5857-0.2906) (Peretto et al. 2013). High angular resolution observations with ALMA towards a large fraction of the infrared-quiet sources of the presented sample indeed reveal a significant population of massive dense cores (Csengeri et al. 2017).

\section{Summary and conclusions}

We investigate the properties of a flux limited sample of massive clumps from the ATLASGAL survey. Based on midinfrared photometry, we divide the sample into infrared-quiet and infrared-bright clumps. Infrared-bright clumps peak on 
T. Csengeri et al.: The ATLASGAL survey: The sample of young massive cluster progenitors
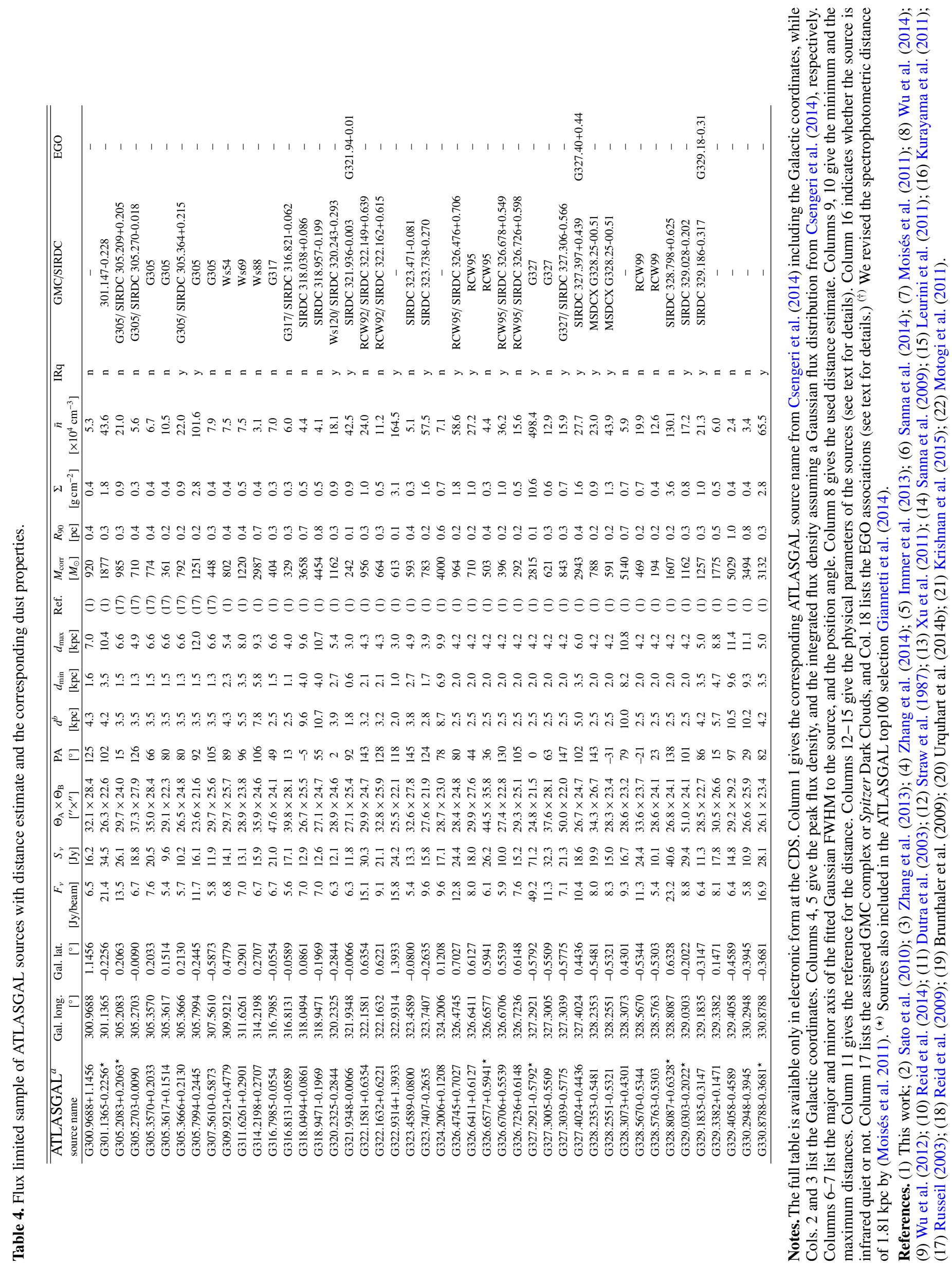

A60, page 13 of 25 


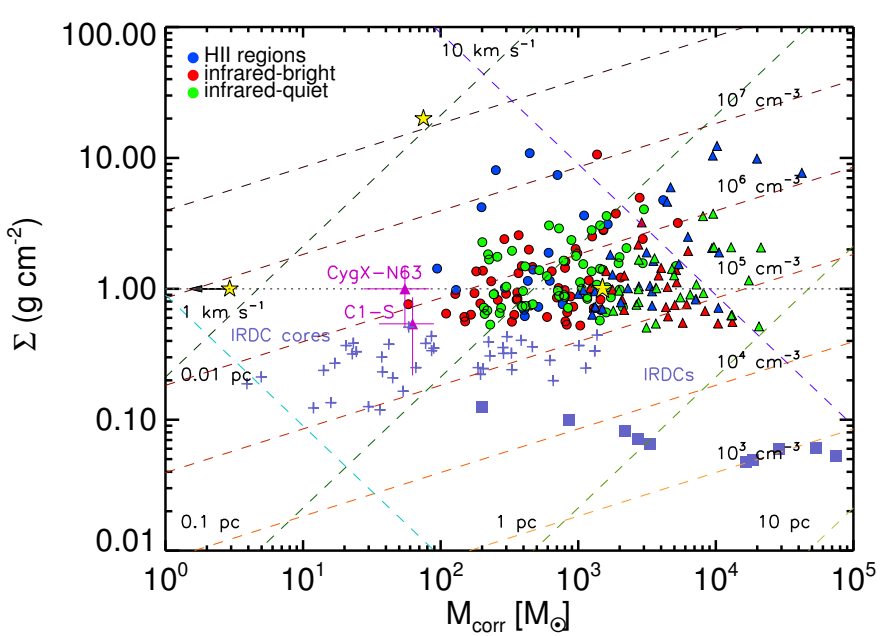

Fig. 13. Surface-density, $\Sigma$, versus temperature corrected mass estimates (using $T_{\mathrm{d}}=30 \mathrm{~K}$ for the infrared-bright clumps). The coloured dashed lines in different shades show constant radius (green), $n_{\mathrm{H}}$ number density (red), and escape speed (blue; see Tan et al. 2014) Colours and symbols are the same as in Fig. 8. We mark two massive cores with $M_{\mathrm{MDC}}=60 M_{\odot}\left(\mathrm{C} 1-\mathrm{S}\right.$; Tan et al. 2013) and $55 M_{\odot}$ (CygX-N63; Bontemps et al. 2010). For comparison IRDC clumps (Kainulainen \& Tan 2013) and cores are shown (Butler \& Tan 2012) The dotted line marks the $1 \mathrm{~g} \mathrm{~cm}^{-3}$ value of Krumholz \& McKee (2008).

embedded objects with $L_{\text {bol }}>10^{4} L_{\odot}$ corresponding to MYSOs or high-mass stars with UC-H II regions, while infrared-quiet clumps lack such bright embedded objects. From the initial sample of 210 sources above a $5 \mathrm{Jy}$ beam-averaged peak flux density, we identify 80 infrared-quiet massive clumps, of which 50 are located within $d<4.5 \mathrm{kpc}$. About one-fourth of them have not been recognised by other surveys.

We estimate their physical properties based on the dust emission, and find that statistically both samples exhibit similar properties in terms of mass and size. Only their embedded (proto)stellar content is different, the infrared-quiet sample lacks luminous embedded objects. This suggests that infrared-quiet clumps are the precursors of the bright clumps, and thus they likely follow the same evolutionary path. A substantial fraction of infrared-quiet clumps exhibit signatures of ongoing star formation activity such as emission from shocked gas associated with material ejection. In particular, selective star formation tracers, such as EGO activity and Class II methanol maser emission, suggest that at least some infrared-quiet massive clumps host high-mass protostars. This makes them excellent candidates to be the potential precursors of the most massive stars forming in our Galaxy.

The overall low ratio of virial mass to mass of the sample suggests a collapse on the clump scale, which is consistent with a fast, dynamic star formation process. In this scenario massive clumps start to fragment and collapse before their final mass is accumulated indicating that strong self-gravity and global collapse is needed to build up rich clusters and the most massive stars.

High angular-resolution observations of infrared-quiet massive clumps are the next step to reveal their protostellar content and uncover the initial conditions for high-mass star formation.

Acknowledgements. We thank the referee for the careful reading of the manuscript. This work was partially funded by the ERC Advanced Investigator Grant GLOSTAR (247078). T.Cs. acknowledges support from the Deutsche Forschungsgemeinschaft, DFG via the SPP (priority programme) 1573 "Physics of the ISM". T.Cs. thanks R. Gutermuth for making available the PhotVis tool. This paper is based on data acquired with the Atacama Pathfinder
EXperiment (APEX). APEX is a collaboration between the Max Planck Institute for Radioastronomy, the European Southern Observatory, and the Onsala Space Observatory. This research made use of data products from the Midcourse Space Experiment. Processing of the data was funded by the Ballistic Missile Defense Organization with additional support from NASA Office of Space Science. This research has also made use of the NASA/IPAC Infrared Science Archive, which is operated by the Jet Propulsion Laboratory, California Institute of Technology, under contract with the National Aeronautics and Space Administration. This publication makes use of data products from the Wide-field Infrared Survey Explorer, which is a joint project of the University of California, Los Angeles, and the Jet Propulsion Laboratory/California Institute of Technology, funded by the National Aeronautics and Space Administration. This research made use of Montage, funded by the National Aeronautics and Space Administration's Earth Science Technology Office, Computation Technologies Project, under Cooperative Agreement Number NCC5-626 between NASA and the California Institute of Technology. Montage is maintained by the NASA/IPAC Infrared Science Archive.

\section{References}

Avison, A., Peretto, N., Fuller, G. A., et al. 2015, A\&A, 577, A30 Bally, J., Anderson, L. D., Battersby, C., et al. 2010, A\&A, 518, L90 Battersby, C., Bally, J., Ginsburg, A., et al. 2011, A\&A, 535, A128 Beltrán, M. T., Sánchez-Monge, Á., Cesaroni, R., et al. 2014, A\&A, 571, A52 Benjamin, R. A., Churchwell, E., Babler, B. L., et al. 2003, PASP, 115, 953 Bergin, E. A., \& Tafalla, M. 2007, ARA\&A, 45, 339 Bernard, J.-P., Paradis, D., Marshall, D. J., et al. 2010, A\&A, 518, L88 Bertoldi, F., \& McKee, C. F. 1992, ApJ, 395, 140

Beuther, H., Zhang, Q., Greenhill, L. J., et al. 2004, ApJ, 616, L31 Bohlin, R. C., Savage, B. D., \& Drake, J. F. 1978, ApJ, 224, 132 Bonnell, I. A., \& Bate, M. R. 2006, MNRAS, 370, 488

Bonnell, I. A., Bate, M. R., Clarke, C. J., \& Pringle, J. E. 2001, MNRAS, 323, 785

Bontemps, S., Motte, F., Csengeri, T., \& Schneider, N. 2010, A\&A, 524, A18 Brand, J., \& Blitz, L. 1993, A\&A, 275, 67

Breen, S. L., Fuller, G. A., Caswell, J. L., et al. 2015, MNRAS, 450, 4109 Bronfman, L., Nyman, L.-A., \& May, J. 1996, A\&AS, 115, 81 Butler, M. J., \& Tan, J. C. 2012, ApJ, 754, 5

Carey, S. J., Noriega-Crespo, A., Mizuno, D. R., et al. 2009, PASP, 121, 76 Caswell, J. L., Fuller, G. A., Green, J. A., et al. 2010, MNRAS, 404, 1029 Caswell, J. L., Fuller, G. A., Green, J. A., et al. 2011, MNRAS, 417, 1964 Chen, X., Gan, C.-G., Ellingsen, S. P., et al. 2013, ApJS, 206, 9 Churchwell, E. 2002, ARA\&A, 40, 27

Csengeri, T., Bontemps, S., Schneider, N., Motte, F., \& Dib, S. 2011a, A\&A, 527, A135

Csengeri, T., Bontemps, S., Schneider, N., et al. 2011b, ApJ, 740, L5 Csengeri, T., Urquhart, J. S., Schuller, F., et al. 2014, A\&A, 565, A75 Csengeri, T., Leurini, S., Wyrowski, F., et al. 2016a, A\&A, 586, A149 Csengeri, T., Weiss, A., Wyrowski, F., et al. 2016b, A\&A, 585, A104 Csengeri, T., Bontemps, S., Wyrowski, F., et al. 2017, A\&A, 600, L10 Cutri, R. M., Wright, E. L., Conrow, T., et al. 2012, Explanatory Supplement to the WISE All-Sky Data Release Products, Tech. rep.

Cyganowski, C. J., Whitney, B. A., Holden, E., et al. 2008, AJ, 136, 2391 Cyganowski, C. J., Brogan, C. L., Hunter, T. R., et al. 2014, ApJ, 796, L2 Da Rio, N., Robberto, M., Hillenbrand, L. A., Henning, T., \& Stassun, K. G. 2012, ApJ, 748, 14

Draine, B. T. 2011, Physics of the Interstellar and Intergalactic Medium (Princeton University Press)

Duarte-Cabral, A., Bontemps, S., Motte, F., et al. 2013, A\&A, 558, A125 Dunham, M. K., Rosolowsky, E., Evans, II, N. J., Cyganowski, C., \& Urquhart, J. S. 2011, ApJ, 741, 110

Dutra, C. M., Bica, E., Soares, J., \& Barbuy, B. 2003, A\&A, 400, 533

Elia, D., Molinari, S., Fukui, Y., et al. 2013, ApJ, 772, 45

Engelbracht, C. W., Blaylock, M., Su, K. Y. L., et al. 2007, PASP, 119, 994

Galván-Madrid, R., Liu, H. B., Zhang, Z.-Y., et al. 2013, ApJ, 779, 121

Garay, G., Faúndez, S., Mardones, D., et al. 2004, ApJ, 610, 313

Giannetti, A., Wyrowski, F., Brand, J., et al. 2014, A\&A, 570, A65

Ginsburg, A., Bally, J., Battersby, C., et al. 2015, A\&A, 573, A106

Green, J. A., Caswell, J. L., Fuller, G. A., et al. 2010, MNRAS, 409, 913

Güsten, R., Nyman, L. A., Schilke, P., et al. 2006, A\&A, 454, L13

Gutermuth, R. A., Myers, P. C., Megeath, S. T., et al. 2008, ApJ, 674, 336

Guzmán, A. E., Garay, G., \& Brooks, K. J. 2010, ApJ, 725, 734

Habibi, M., Stolte, A., Brandner, W., Hußmann, B., \& Motohara, K. 2013, A\&A, 556, A 26

Hoare, M. G., \& Franco, J. 2007, Astrophys. Space Sci. Proc., 1, 61

Hoare, M. G., Kurtz, S. E., Lizano, S., Keto, E., \& Hofner, P. 2007, Protostars and Planets V, 181 
T. Csengeri et al.: The ATLASGAL survey: The sample of young massive cluster progenitors

Hoare, M. G., Purcell, C. R., Churchwell, E. B., et al. 2012, PASP, 124, 939 Hunter, T. R., Churchwell, E., Watson, C., et al. 2000, AJ, 119, 2711

Immer, K., Reid, M. J., Menten, K. M., Brunthaler, A., \& Dame, T. M. 2013, A\&A, 553, A117

Jackson, J. M., Rathborne, J. M., Shah, R. Y., et al. 2006, ApJS, 163, 145

Jackson, J. M., Finn, S. C., Rathborne, J. M., Chambers, E. T., \& Simon, R. 2008, ApJ, 680, 349

Kainulainen, J., \& Tan, J. C. 2013, A\&A, 549, A53

Kauffmann, J., \& Pillai, T. 2010, ApJ, 723, L7

Kauffmann, J., Bertoldi, F., Bourke, T. L., Evans, II, N. J., \& Lee, C. W. 2008 , A\&A, 487, 993

Kauffmann, J., Pillai, T., Shetty, R., Myers, P. C., \& Goodman, A. A. 2010, ApJ, 712,1137

Kauffmann, J., Pillai, T., \& Goldsmith, P. F. 2013, ApJ, 779, 185

Keto, E. 2002, ApJ, 580, 980

Keto, E. 2003, ApJ, 599, 1196

Klaassen, P. D., Testi, L., \& Beuther, H. 2012, A\&A, 538, A140

Klein, T., Ciechanowicz, M., Leinz, C., et al. 2014, IEEE Transactions on Terahertz Science and Technology, 4, 588

König, C., Urquhart, J. S., Csengeri, T., et al. 2017, A\&A, 599, A139

Krishnan, V., Ellingsen, S. P., Reid, M. J., et al. 2015, ApJ, 805, 129

Krumholz, M. R., \& McKee, C. F. 2008, Nature, 451, 1082

Kuiper, R., \& Yorke, H. W. 2013, ApJ, 772, 61

Kurayama, T., Nakagawa, A., Sawada-Satoh, S., et al. 2011, PASJ, 63, 513

Leurini, S., Codella, C., Zapata, L., et al. 2011, A\&A, 530, A12

Leurini, S., Codella, C., López-Sepulcre, A., et al. 2014, A\&A, 570, A49

López, C., Bronfman, L., Nyman, L.-Å., May, J., \& Garay, G. 2011, A\&A, 534, A131

Louvet, F., Motte, F., Hennebelle, P., et al. 2014, A\&A, 570, A15

Lumsden, S. L., Hoare, M. G., Urquhart, J. S., et al. 2013, ApJS, 208, 11

McKee, C. F., \& Ostriker, E. C. 2007, ARA\&A, 45, 565

McKee, C. F., \& Tan, J. C. 2003, ApJ, 585, 850

Megeath, S. T., \& Tieftrunk, A. R. 1999, ApJ, 526, L113

Megeath, S. T., Gutermuth, R., Muzerolle, J., et al. 2012, AJ, 144, 192

Menten, K. M. 1991, ApJ, 380, L75

Moisés, A. P., Damineli, A., Figuerêdo, E., et al. 2011, MNRAS, 411, 705

Molinari, S., Brand, J., Cesaroni, R., \& Palla, F. 1996, A\&A, 308, 573

Molinari, S., Brand, J., Cesaroni, R., \& Palla, F. 2000, A\&A, 355, 617

Molinari, S., Pezzuto, S., Cesaroni, R., et al. 2008, A\&A, 481, 345

Mookerjea, B., Kramer, C., Nielbock, M., \& Nyman, L.-A. 2004, A\&A, 426, 119

Motogi, K., Sorai, K., Habe, A., et al. 2011, PASJ, 63, 31

Motte, F., Schilke, P., \& Lis, D. C. 2003, ApJ, 582, 277

Motte, F., Bontemps, S., Schilke, P., et al. 2007, A\&A, 476, 1243

Mottram, J. C., Hoare, M. G., Davies, B., et al. 2011, ApJ, 730, L33

Mufson, S. L., \& Liszt, H. S. 1979, ApJ, 232, 451

Nguyen Luong, Q., Motte, F., Schuller, F., et al. 2011, A\&A, 529, A41

Ossenkopf, V., \& Henning, T. 1994, A\&A, 291, 943

Peng, T.-C., Wyrowski, F., van der Tak, F. F. S., Menten, K. M., \& Walmsley, C. M. 2010, A\&A, 520, A84

Peretto, N., \& Fuller, G. A. 2009, A\&A, 505, 405

Peretto, N., Fuller, G. A., Duarte-Cabral, A., et al. 2013, A\&A, 555, A112

Peretto, N., Lenfestey, C., Fuller, G. A., et al. 2016, A\&A, 590, A72

Purcell, C. R., Hoare, M. G., Cotton, W. D., et al. 2013, ApJS, 205, 1

Rathborne, J. M., Jackson, J. M., Chambers, E. T., et al. 2005, ApJ, 630, L181
Rathborne, J. M., Jackson, J. M., \& Simon, R. 2006, ApJ, 641, 389 Rathborne, J. M., Jackson, J. M., Zhang, Q., \& Simon, R. 2008, ApJ, 689, 1141 Reid, M. J., Menten, K. M., Zheng, X. W., et al. 2009, ApJ, 700, 137 Reid, M. J., Menten, K. M., Brunthaler, A., et al. 2014, ApJ, 783, 130 Russeil, D. 2003, A\&A, 397, 133

Russeil, D., Zavagno, A., Motte, F., et al. 2010, A\&A, 515, A55

Rygl, K. L. J., Brunthaler, A., Sanna, A., et al. 2012, A\&A, 539, A79

Sánchez-Monge, Á., Cesaroni, R., Beltrán, M. T., et al. 2013, A\&A, 552, L10

Sanna, A., Reid, M. J., Moscadelli, L., et al. 2009, ApJ, 706, 464

Sanna, A., Reid, M. J., Menten, K. M., et al. 2014, ApJ, 781, 108

Sato, M., Reid, M. J., Brunthaler, A., \& Menten, K. M. 2010, ApJ, 720, 1055

Schneider, N., Csengeri, T., Bontemps, S., et al. 2010, A\&A, 520, A49

Schuller, F., Menten, K. M., Contreras, Y., et al. 2009, A\&A, 504, 415

Simon, R., Rathborne, J. M., Shah, R. Y., Jackson, J. M., \& Chambers, E. T. 2006, ApJ, 653, 1325

Siringo, G., Kreysa, E., Kovács, A., et al. 2009, A\&A, 497, 945

Sobolev, A. M., Cragg, D. M., \& Godfrey, P. D. 1997, MNRAS, 288, L39

Sridharan, T. K., Beuther, H., Schilke, P., Menten, K. M., \& Wyrowski, F. 2002, ApJ, 566, 931

Sridharan, T. K., Rao, R., Qiu, K., et al. 2014, ApJ, 783, L31

Straw, S., Hyland, A. R., Jones, T. J., et al. 1987, ApJ, 314, 283

Svoboda, B. E., Shirley, Y. L., Battersby, C., et al. 2016, ApJ, 822, 59

Tackenberg, J., Beuther, H., Henning, T., et al. 2014, A\&A, 565, A101

Tackenberg, J., Beuther, H., Henning, T., et al. 2012, A\&A, 540, A113

Tan, J. C., Kong, S., Butler, M. J., Caselli, P., \& Fontani, F. 2013, ApJ, 779, 96

Tan, J. C., Beltrán, M. T., Caselli, P., et al. 2014, Protostars and Planets VI, 149

Tan, J. C., Kong, S., Zhang, Y., et al. 2016, ApJ, 821, L3

Thompson, M. A., Gibb, A. G., Hatchell, J. H., Wyrowski, F., \& Pestalozzi, M. R. 2005, in Protostars and Planets V Posters, 8109

Traficante, A., Fuller, G. A., Peretto, N., Pineda, J. E., \& Molinari, S. 2015, MNRAS, 451, 3089

Urquhart, J. S., Busfield, A. L., Hoare, M. G., et al. 2007, A\&A, 461, 11

Urquhart, J. S., Moore, T. J. T., Schuller, F., et al. 2013a, MNRAS, 431, 1752

Urquhart, J. S., Thompson, M. A., Moore, T. J. T., et al. 2013b, MNRAS, 435, 400

Urquhart, J. S., Moore, T. J. T., Csengeri, T., et al. 2014, MNRAS, 443, 1555

Ward-Thompson, D., André, P., Crutcher, R., et al. 2007, Protostars and Planets $\mathrm{V}, 33$

Wienen, M., Wyrowski, F., Schuller, F., et al. 2012, A\&A, 544, A146

Wienen, M., Wyrowski, F., Menten, K. M., et al. 2015, A\&A, 579, A91

Wienen, M., Wyrowski, F., \& Menten, K. M., et al. 2017, A\&A, submitted

Williams, J. P., Blitz, L., \& McKee, C. F. 2000, Protostars and Planets IV, 97

Wood, D. O. S., \& Churchwell, E. 1989, ApJ, 340, 265

Wright, E. L., Eisenhardt, P. R. M., Mainzer, A. K., et al. 2010, AJ, 140, 1868

Wu, Y. W., Xu, Y., Menten, K. M., Zheng, X. W., \& Reid, M. J. 2012, IAU Symp. 287, eds. R. S. Booth, W. H. T. Vlemmings, \& E. M. L. Humphreys, 425

Wu, Y. W., Sato, M., Reid, M. J., et al. 2014, A\&A, 566, A17

Xu, Y., Moscadelli, L., Reid, M. J., et al. 2011, ApJ, 733, 25

Zapata, L. A., Palau, A., Ho, P. T. P., et al. 2008, A\&A, 479, L25

Zavagno, A., Russeil, D., Motte, F., et al. 2010, A\&A, 518, L81

Zhang, B., Reid, M. J., Menten, K. M., et al. 2013, ApJ, 775, 79

Zhang, B., Moscadelli, L., Sato, M., et al. 2014, ApJ, 781, 89

Zhang, Q., Wang, Y., Pillai, T., \& Rathborne, J. 2009, ApJ, 696, 268

Zinnecker, H., \& Yorke, H. W. 2007, ARA\&A, 45, 481 


\section{Appendix A: Mid-infrared photometry}

\section{A.1. MIPSGAL upper limits}

Using the publicly available calibrated MIPSGAL tiles, we searched for embedded compact objects spatially coinciding with the ATLASGAL dust peaks. From the 219 brightest submm sources we find that 27 do not show any clear point source within 5 " from the dust peaks. After a visual inspection, we extracted an upper limit for these sources by taking the median of the pixel values within a radius of $3^{\prime \prime}$, i.e. half of the FWHM of the PSF at $24 \mu \mathrm{m}$. We then multiply this value by the beam area assuming a Gaussian beam with a FWHM of $6^{\prime \prime}$ to obtain an upper limit on the source flux. We estimate the error by calculating the REDMSQ $^{5}$ value in the same area, in the footprint of the PSF, as was defined in Megeath et al. (2012). We extract upper limits between $0.041-1.368 \mathrm{Jy} /$ beam with an average of $0.4 \mathrm{Jy} / \mathrm{beam}$.

\section{A.2. MIPSGAL aperture photometry at $24 \mu \mathrm{m}$}

We performed aperture photometry towards the dust peaks that are clearly associated with a $24 \mu \mathrm{m}$ point source and without saturated pixels. In greater detail, we extracted the flux density of point sources from the MIPSGAL data using the PhotVis v1.10 tool (Gutermuth et al. 2008) positionally coinciding with the ATLASGAL source. We adopted a zero point magnitude of 12.448 , which was calculated assuming a pixel size of 1 '!25, an aperture correction of 2.05, which is used for apertures of $7 "$ with a sky annulus between 20" to 32" (Engelbracht et al. 2007) ${ }^{6}$. We chose these radii because the background emission varies substantially and a larger sky annulus helps to avoid contamination by nearby bright sources. However, our background values still show variations and so may still be contaminated by extended emission. Therefore, we caution that our values are subject to uncertainties.

We then converted the measured magnitudes to flux density using $F_{v}=\mathrm{c}_{\mathrm{corr}} \times F_{v 0} \times 10^{(-\mathrm{mag} / 2.5)}$, where $F_{\nu 0}$ corresponds to the zero magnitude flux level, which is $7.17 \mathrm{Jy}$ and mag is the magnitude given by PhtoVis. The targeted sample consists of deeply embedded objects, where $\beta \sim 1-2$ corresponds to rising SEDs towards longer wavelengths. Therefore, we applied a colour correction ( $c_{\text {corr }}$ ) of 0.981 for objects with $\beta \sim 1$ (Engelbracht et al. 2007).

\section{A.3. Comparing MIPSGAL and WISE flux densities}

We measure $24 \mu \mathrm{m}$ flux densities (and upper limits) between 0.041 and $6.88 \mathrm{Jy}$ for these sources, with a mean of 1.84 and a median of $0.81 \mathrm{Jy}$. For 75 sources in the sample we also have

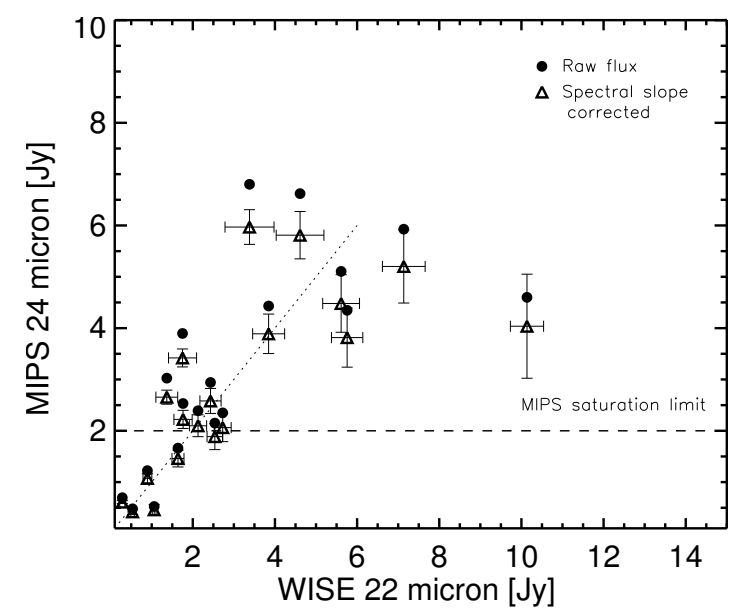

Fig. A.1. Cross-calibration between flux densities from the WISE point source catalogue and the MIPSGAL survey.

photometry from the WISE point source catalogue (Wright et al. 2010). Since there is an overlap in the wavelength ranges of the WISE band at $22 \mu \mathrm{m}$ and the MIPS detector at $24 \mu \mathrm{m}$, we attempted to cross-calibrate the photometry of the two measurements. We found that numerous sources appearing in the WISE catalogue and not saturated with MIPS are flagged as likely spurious detections. This is likely due to source confusion and extended nebulosity surrounding the majority of our weak sources, which results in bad quality flags for these sources in the WISE point source catalogue. Therefore, we only have 21 sources with reliable flux measurement from WISE, of which only 19 have smaller than $5^{\prime \prime}$ angular offset from the ATLASGAL peak position.

Our attempt to cross-calibrate the two instruments is shown in Fig. A.1, where we also correct for a spectral index of 1.5 that has been found between MSX and MIPSGAL according to the MIPSGAL data delivery document ${ }^{7}$. With some scatter, we find similar flux density measurements below $\sim 4$ Jy with the two instruments. Since the MIPS detector starts to saturate at $2 \mathrm{Jy}$, the flattening of the MIPSGAL fluxes between $\sim 2-4$ Jy is likely due to the saturation or non-linearity of the detector. In addition, since WISE has a beam that is two times larger (the MIPS PSF is $6^{\prime \prime}$ at $24 \mu \mathrm{m}$, while WISE has a $12^{\prime \prime}$ angular resolution), it may include emission from the surrounding diffuse emission, not only the protostar. Since saturation and non-linearity of the detector may significantly influence the determined values, in our analysis for sources above $2 \mathrm{Jy}$ we rely on the WISE point source catalogue values.

\footnotetext{
$5 \operatorname{REDMSQ}=\sqrt{\operatorname{median}\left(\left(S[i, j]-\operatorname{median}(S[i, j])^{2}\right)\right.}$.

6 We used the following formula to calculate the zero point magnitude, $\operatorname{mag}_{0}=2.5 \times \log _{10}\left(\frac{F_{v 0}}{10^{6} \times \Omega \times \text { app }_{\text {corr }}}\right)$, where $\Omega$ corresponds to the pixel size in steradian and $F_{v 0}$ is $7.17 \mathrm{Jy}$.
}

\footnotetext{
7 http://irsa.ipac.caltech.edu/data/SPITZER/MIPSGAL/ images/mipsgal_delivery_guide_v3_29aug08.pdf
} 
T. Csengeri et al.: The ATLASGAL survey: The sample of young massive cluster progenitors

\section{Appendix B: Infrared-quiet massive clumps}
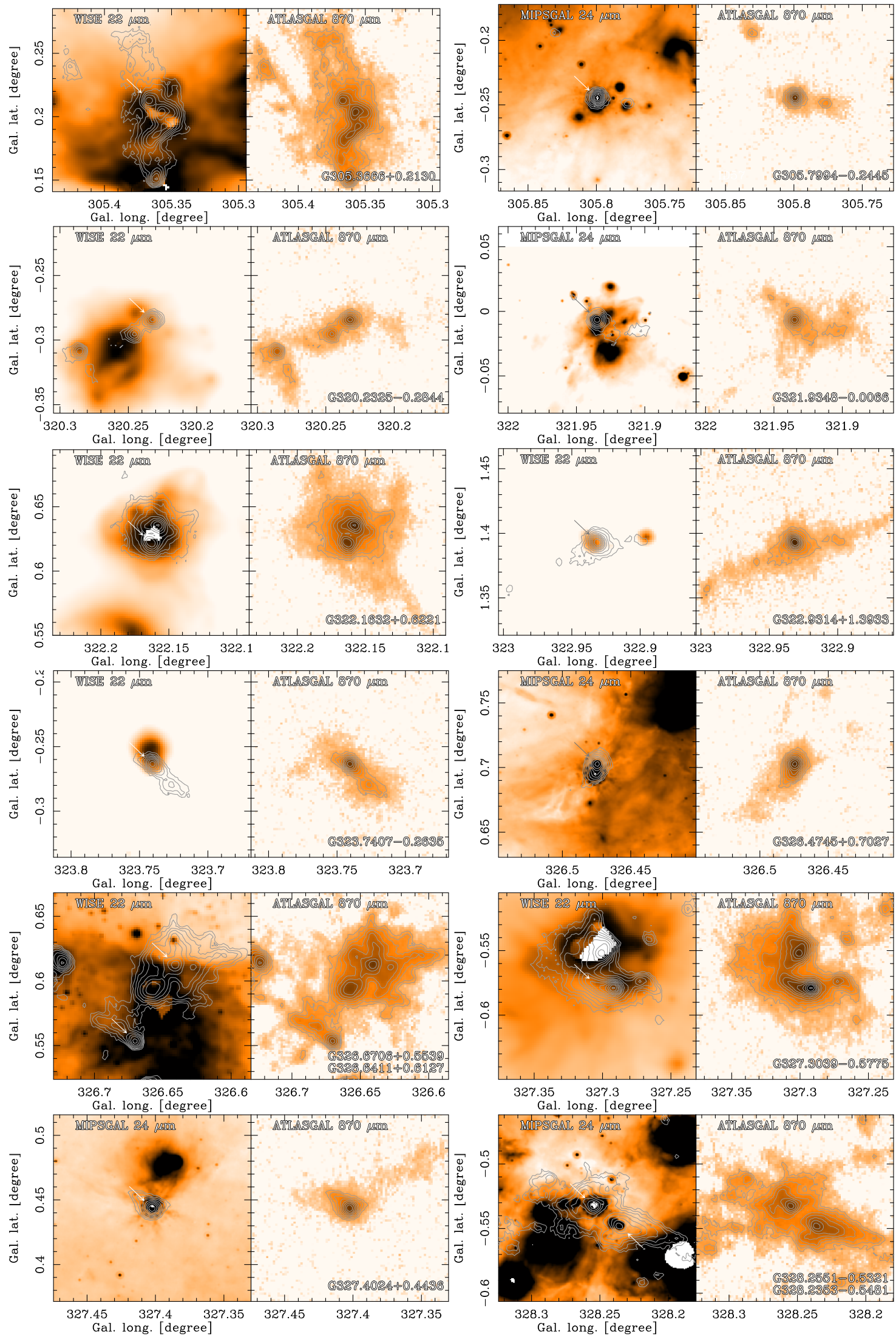

Fig. B.1. Left: mid-infrared emission from MIPSGAL at $24 \mu \mathrm{m}$ or WISE at $22 \mu \mathrm{m}$ in colour scale with the $870 \mu \mathrm{m}$ contours from ATLASGAL. The arrows mark the position of infrared-quiet clumps. Right: $870 \mu \mathrm{m}$ emission with contours from ATLASGAL. The blue crosses mark the positions of UC-H II regions from CORNISH. The figure label shows the corresponding ATLASGAL source name. 
A\&A 601, A60 (2017)
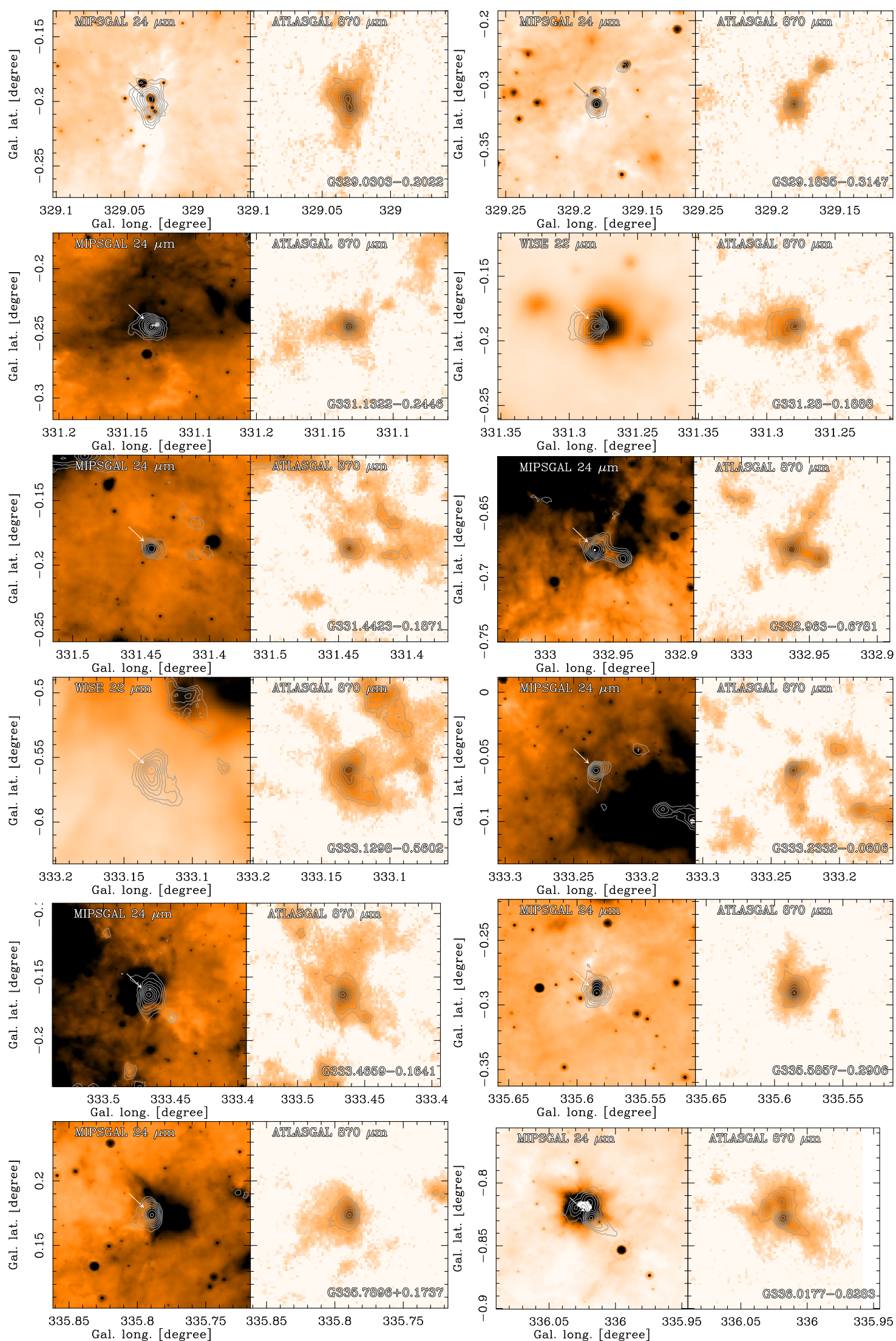

Fig. B.1. continued. 
T. Csengeri et al.: The ATLASGAL survey: The sample of young massive cluster progenitors
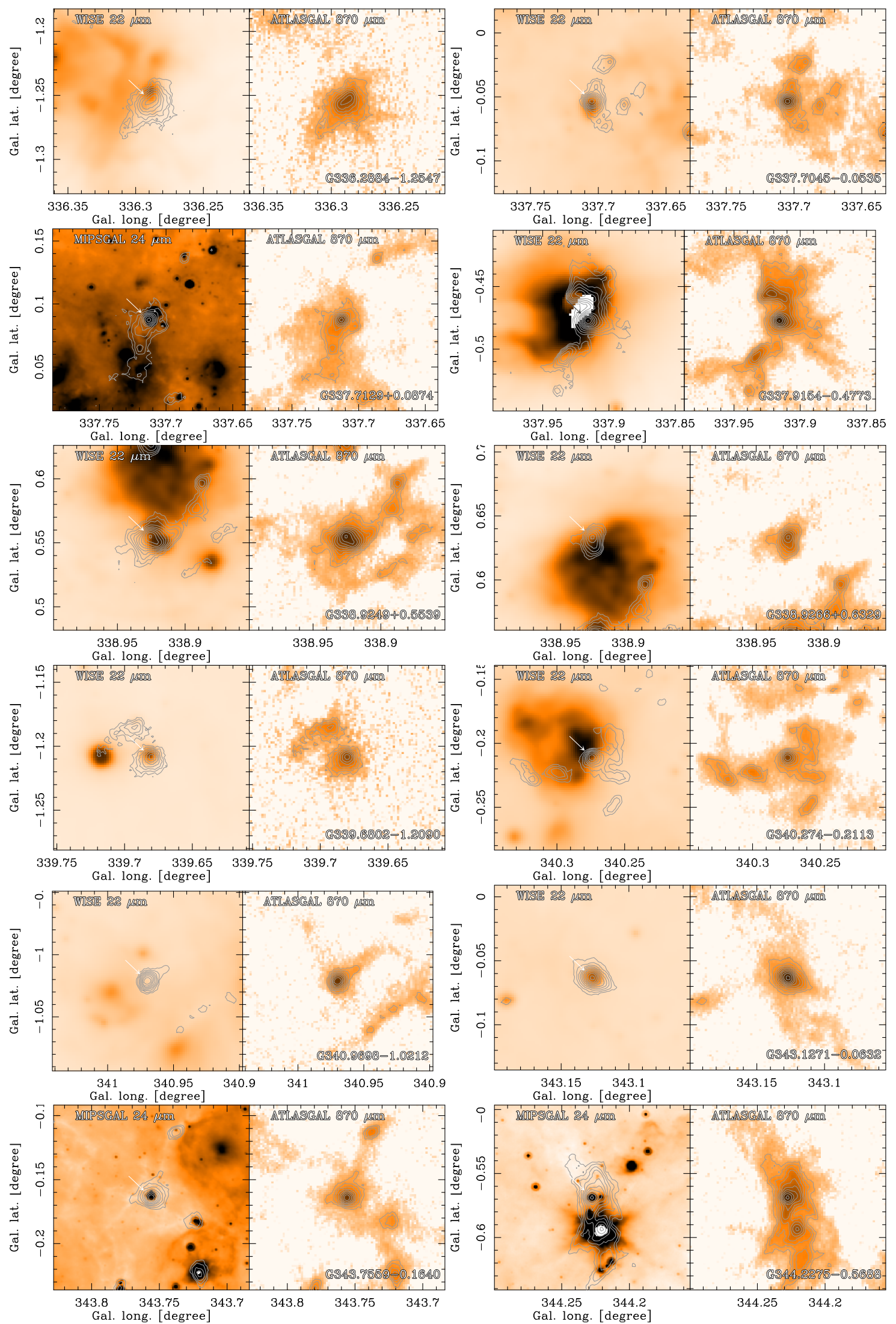

Fig. B.1. continued. 
A\&A 601, A60 (2017)
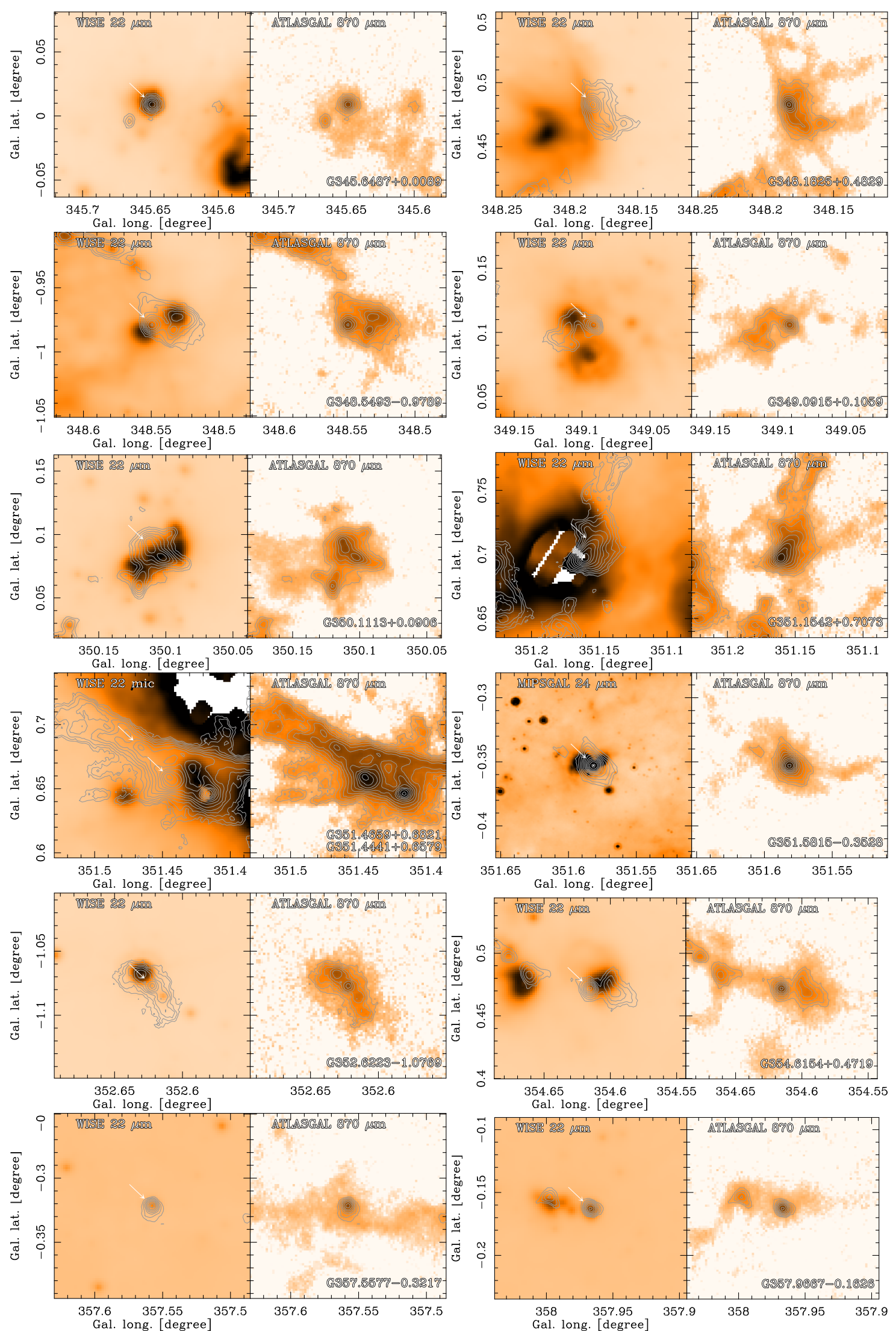

Fig. B.1. continued. 
T. Csengeri et al.: The ATLASGAL survey: The sample of young massive cluster progenitors
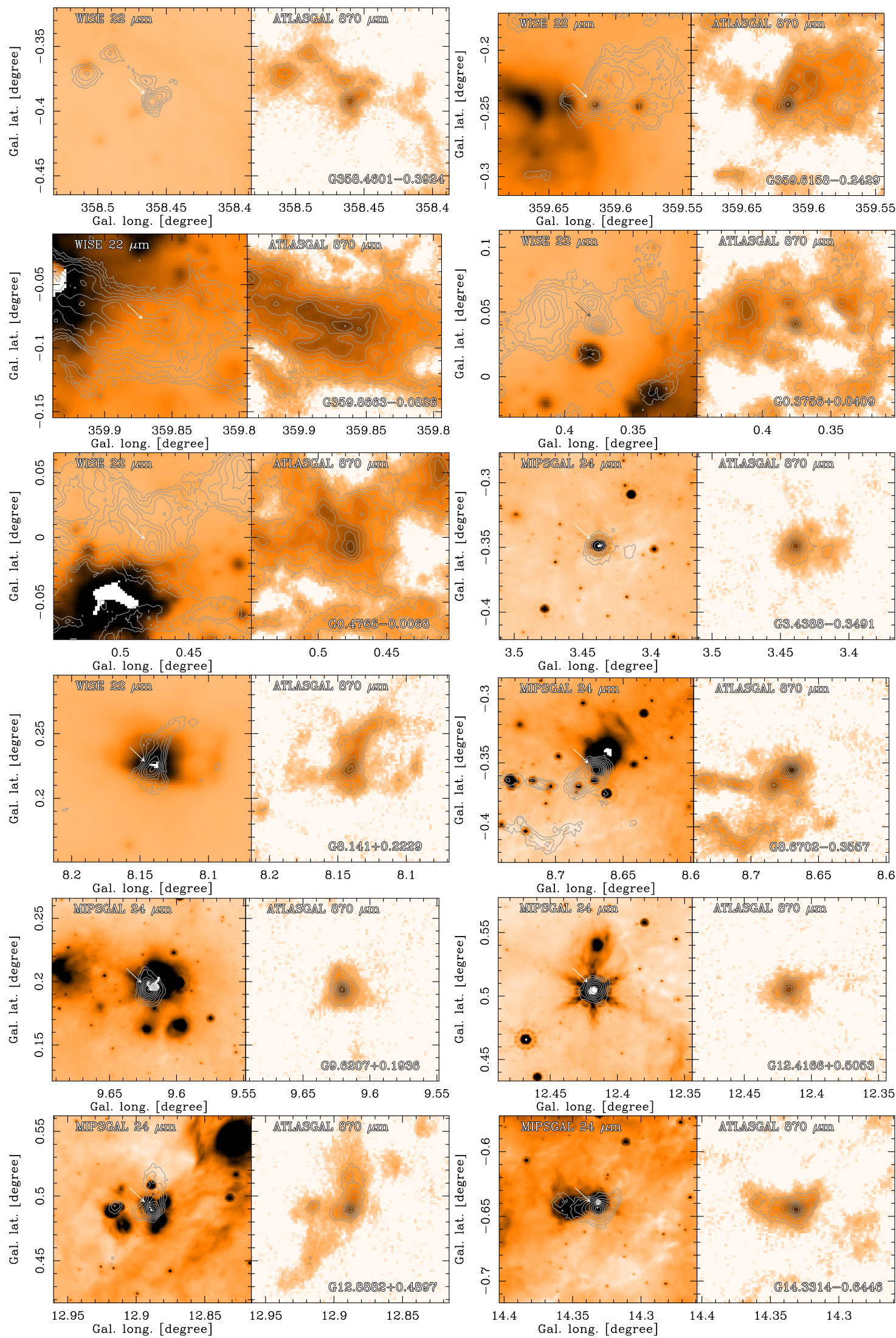

Fig. B.1. continued. 
A\&A 601, A60 (2017)
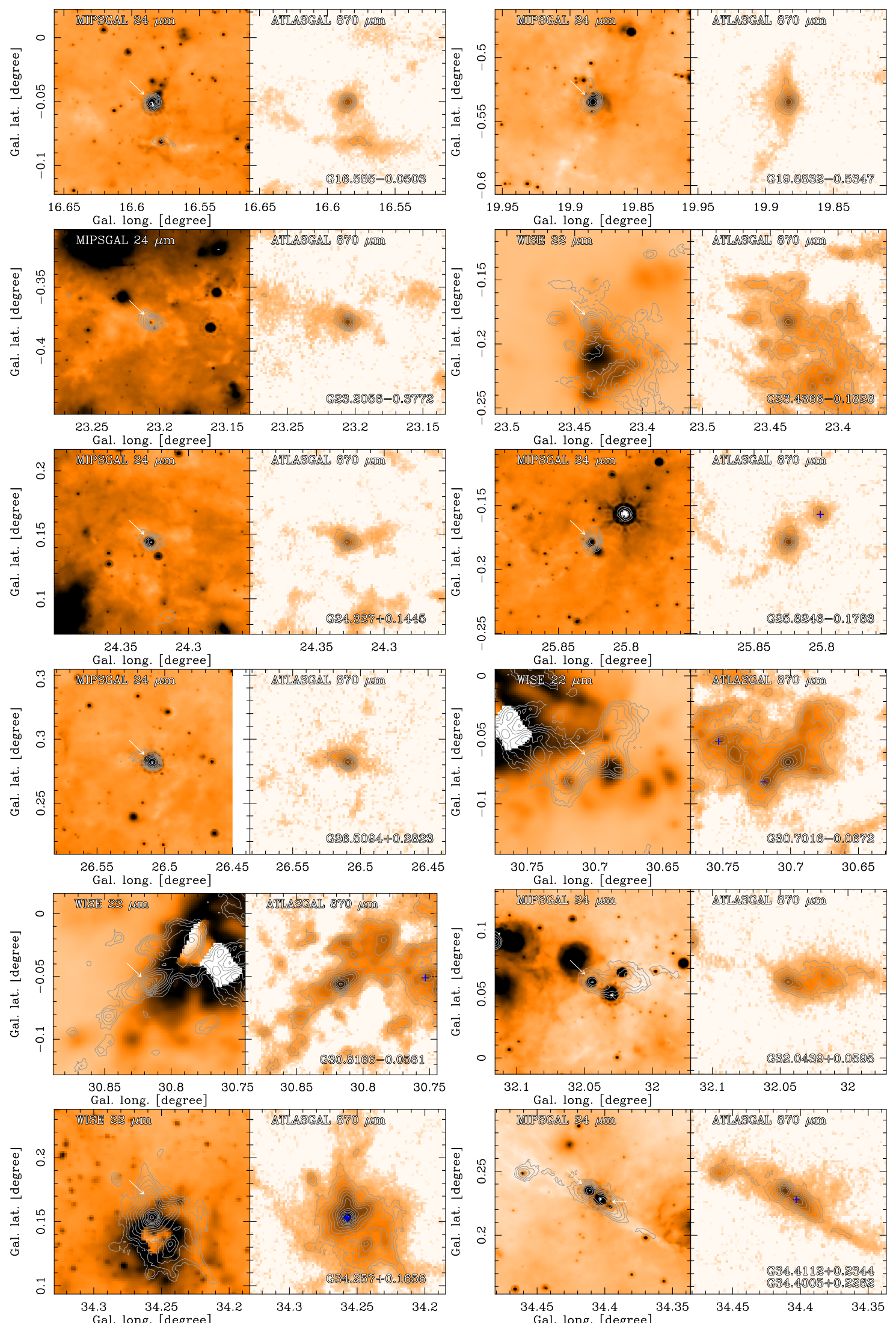

Fig. B.1. continued. 
T. Csengeri et al.: The ATLASGAL survey: The sample of young massive cluster progenitors
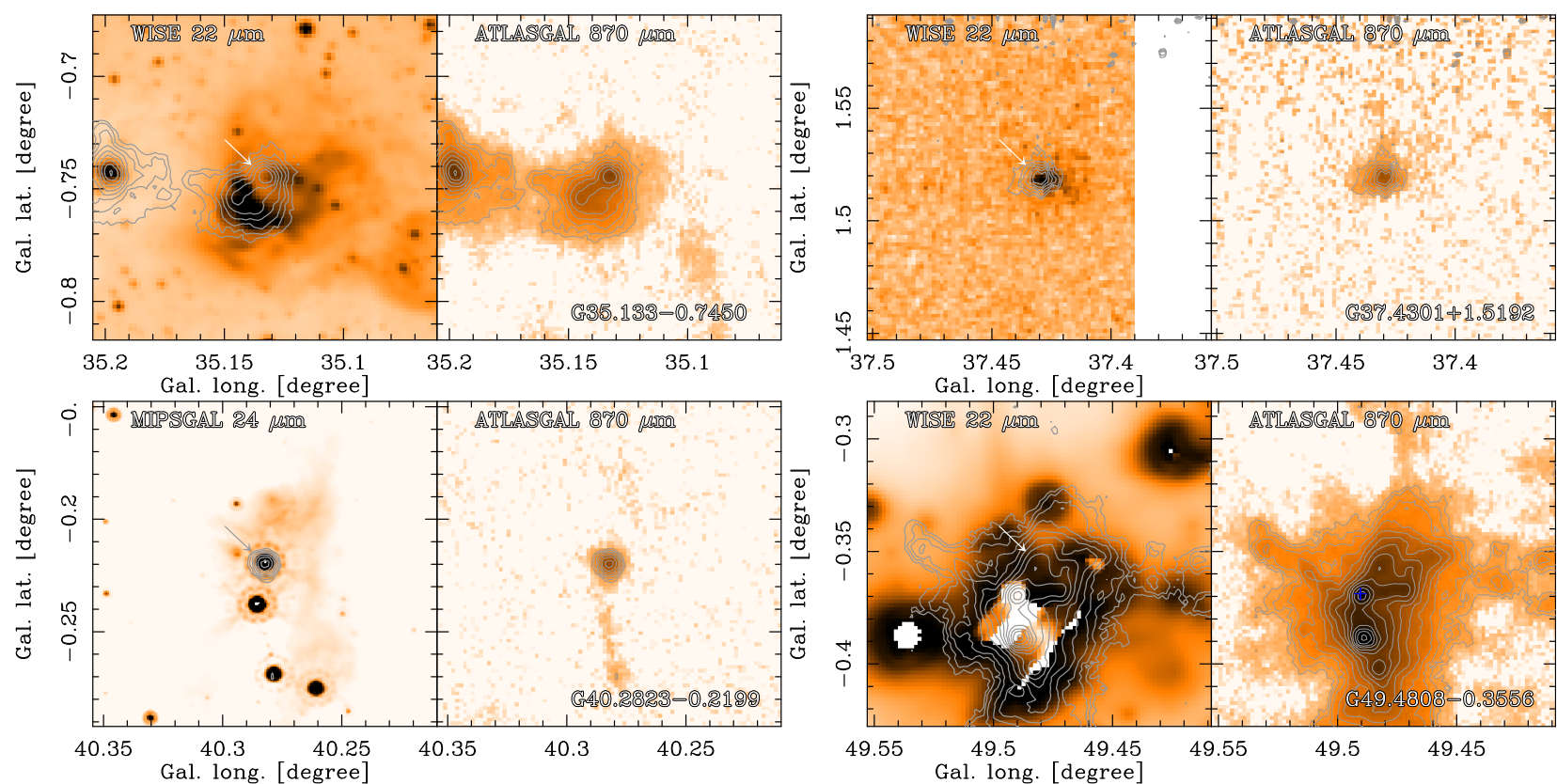

Fig. B.1. continued. 


\section{Appendix C: SiO (8-7) lines towards infrared-quiet massive clumps with APEX}
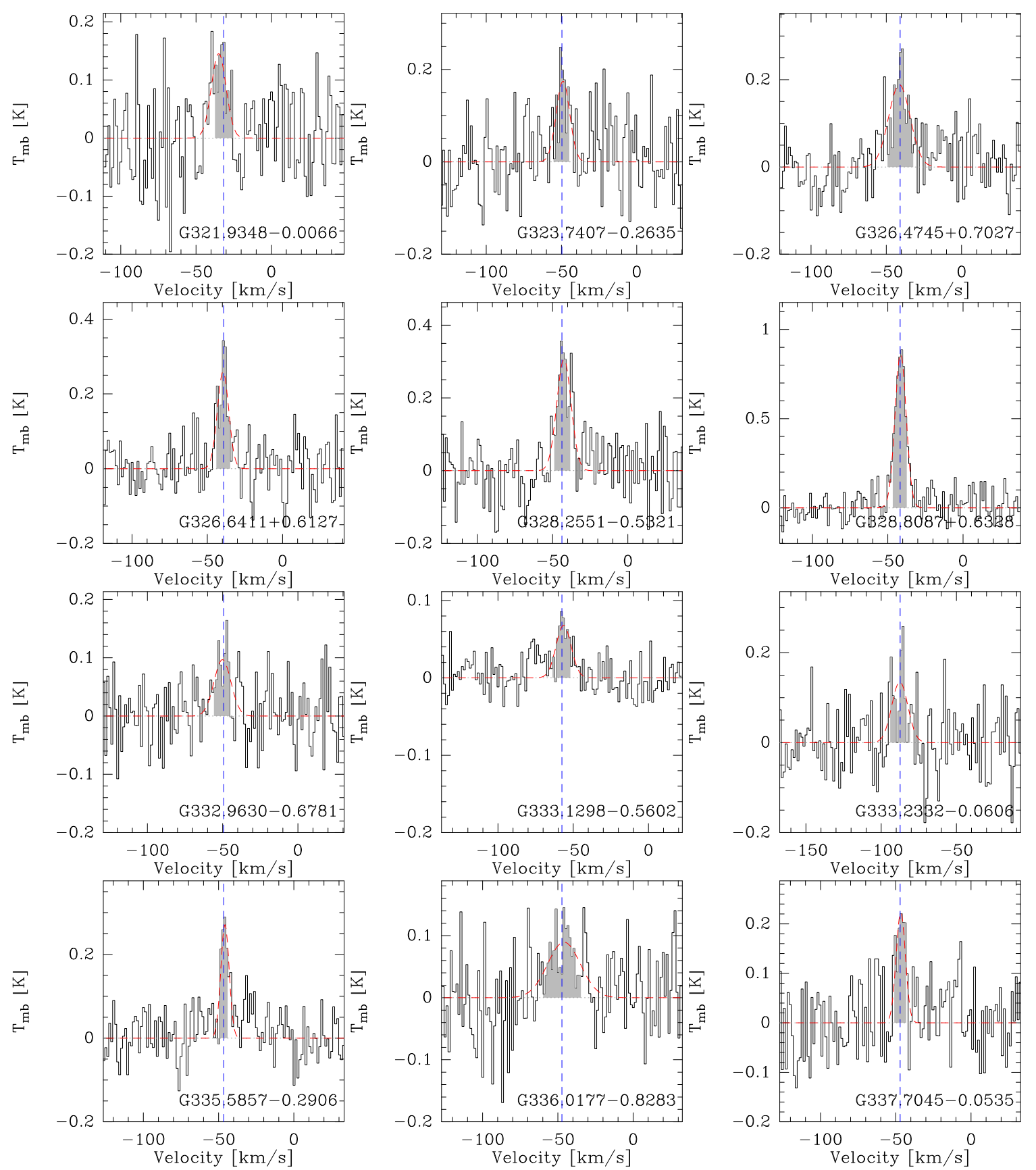

Fig. C.1. SiO (8-7) detections with the APEX/FLASH ${ }^{+}$receiver of a selection of infrared-quiet massive clumps. The red dashed line shows the fitted Gaussians, and the grey histogram shows the line area. The blue dashed line indicates the $v_{\mathrm{lsr}}$ of the sources. 
T. Csengeri et al.: The ATLASGAL survey: The sample of young massive cluster progenitors
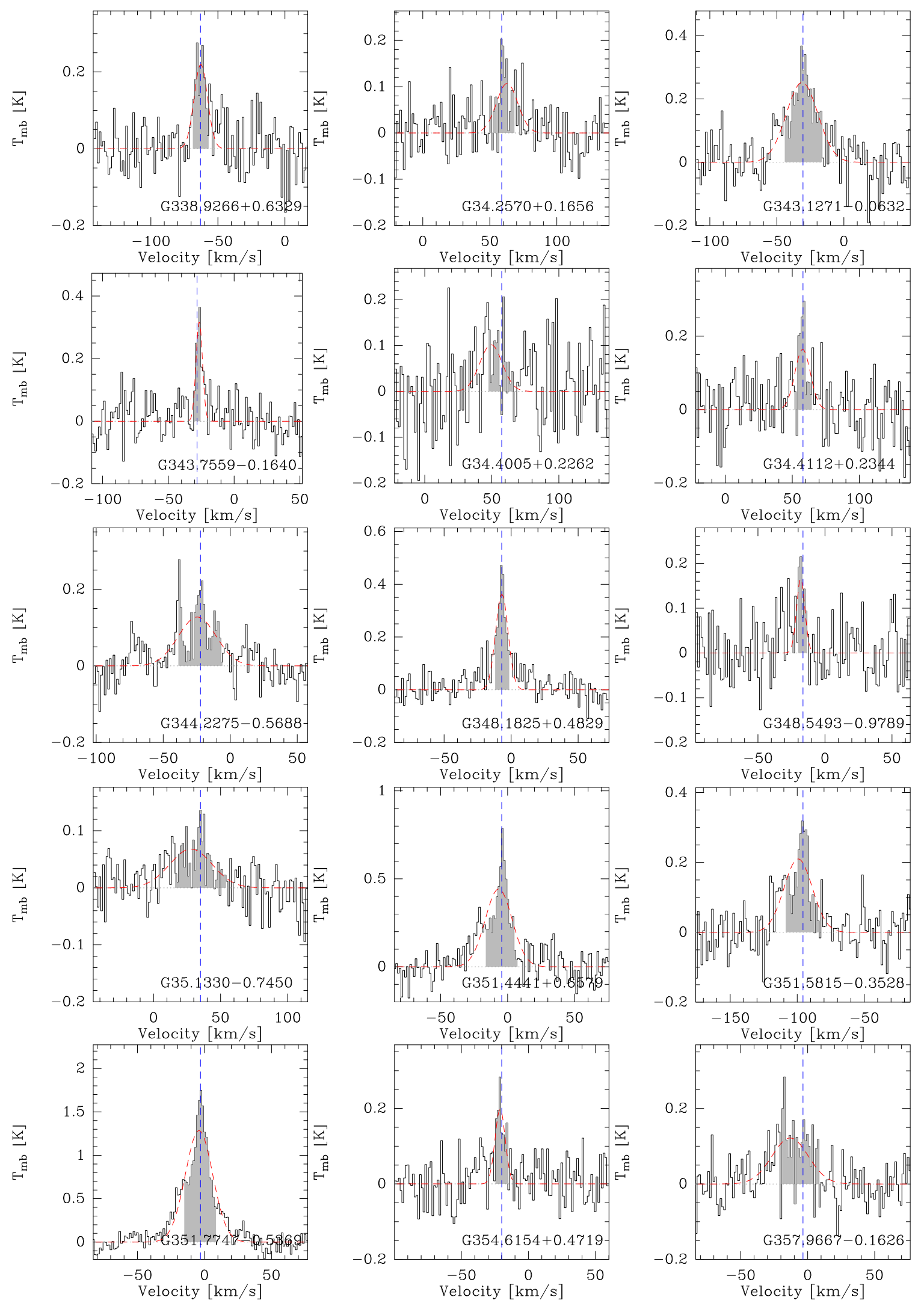

Fig. C.1. continued. 